\title{
Gene expression profiling during adventitious root formation in carnation stem cuttings
}

\author{
Carlos Villacorta-Martín ${ }^{1}$, Ana Belén Sánchez-García², Joan Villanova², Antonio Cano ${ }^{3}$, Miranda van de Rhee ${ }^{1}$ \\ Jorn de Haan ${ }^{1}$, Manuel Acosta ${ }^{3}$, Paul Passarinho ${ }^{1}$ and José Manuel Pérez-Pérez ${ }^{2 *}$
}

\begin{abstract}
Background: Adventitious root (AR) formation is a critical step in vegetative propagation of most ornamental plants, such as carnation. AR formation from stem cuttings is usually divided into several stages according to physiological and metabolic markers. Auxin is often applied exogenously to promote the development of ARs on stem cuttings of difficult-to-root genotypes.

Results: By whole transcriptome sequencing, we identified the genes involved in AR formation in carnation cuttings and in response to exogenous auxin. Their expression profiles have been analysed through RNA-Seq during a time-course experiment in the stem cutting base of two cultivars with contrasting efficiencies of AR formation. We explored the kinetics of root primordia formation in these two cultivars and in response to exogenously-applied auxin through detailed histological and physiological analyses.

Conclusions: Our results provide, for the first time, a number of molecular, histological and physiological markers that characterize the different stages of AR formation in this species and that could be used to monitor adventitious rooting on a wide collection of carnation germplasm with the aim to identify the best-rooting cultivars for breeding purposes.
\end{abstract}

Keywords: RNA-Seq, Time-series analysis, Differential expression profiling, Regulatory network analysis, Dianthus caryophyllus

\section{Background}

In horticulture and forestry, vegetative propagation is widely used for the multiplication of plants with optimal phenotypes obtained in breeding programs or selected from natural populations. Adventitious root (AR) formation is a critical step in vegetative propagation: substantial losses can occur because cuttings do not form roots or they form poor quality root systems. A conservative estimation quantifies the burden of inadequate rooting treatments on US \$50 million per year only in The Netherlands.

ARs are distinct from lateral roots in that they form from any tissue that is not a root, such as leaves and stems, naturally or in response to altered environments $[1,2]$. AR formation from stem cuttings is usually divided into several stages according to physiological and metabolic markers: $i$ ) dedifferentiation, during which cells become competent to respond to the rhizogenic signal (auxin), ii) cell division (or induction phase), and iii) root

\footnotetext{
* Correspondence: jmperez@umh.es

${ }^{2}$ Instituto de Bioingeniería, Universidad Miguel Hernández, 03202 Elche,

Alicante, Spain

Full list of author information is available at the end of the article
}

primordia outgrowth from the stem [3]. Several plant hormones are known to control AR formation, of which auxin is a central player [4]. Auxin is often applied exogenously to promote the development of ARs on stem cuttings of difficult-to-root genotypes [1, 3]. In many species, high auxin levels in the basal region of the cuttings are required for the competent cells in the cambium to resume proliferation and to start the root-specific developmental program $[5,6]$. Consistently with a positive role for auxin in AR formation, Arabidopsis mutants overproducing auxin spontaneously develop ARs on the hypocotyl [7-9]. Auxin and cytokinins are known to play a crucial role in many aspects of plant development, often acting antagonistically. A negative role for cytokinins in AR formation has been proposed as mutants defective in cytokinin biosynthesis or perception displayed increased production of ARs, whereas enhanced cytokinin biosynthesis has the opposite effect [10-12]. Moreover, interrelationships between auxin and carbohydrate metabolism during adventitious rooting have been investigated by the application of exogenous auxins and by monitoring of carbohydrate levels, carbon translocation and activities 
of key enzymes involved in sugar metabolism in the rooting zone [13-15].

Various molecular approaches have been employed to study AR development in Arabidopsis and other model plants [1]. In Arabidopsis, it was shown that the balance between AUXIN RESPONSE FACTOR17 (ARF17), a negative regulator of adventitious rooting, and ARF6 and ARF8, positive regulators of AR formation, as well as the maintenance of the homeostasis of their regulatory microRNAs (miRNAs), plays a critical role in AR formation [16, 17]. Additionally, the proteomic analysis of mutants affected in AR formation led to the identification of 11 proteins, including three auxin-inducible GRETCHEN HAGEN (GH3)-like proteins, whose expression was altered [18]. In turn, these GH3-like proteins are required for fine-tuning AR initiation in the hypocotyl, through modulating jasmonic acid homeostasis [19]. These results suggest that the early stages of AR formation need to be tightly regulated at the physiological and the genetic level and that improving rooting performance of economically important genotypes requires identifying the molecular components of the hormonal crosstalk that regulates AR formation in nonmodel species. As an alternative strategy to identify genes involved in AR formation, a number of studies have been conducted to characterize the gene expression profiles in the stem cutting base of different species during rooting [13, 20-25]. Based on these studies, some of the molecular events occurring during AR formation have been outlined. In our study, we aimed to characterize gene expression and functional changes occurring in the stem cutting base during the early stages of adventitious rooting in two carnation cultivars, $2003 R 8$ and 2101-02 MFR, which have been selected because of their contrasting rooting performance [26]. Our results will allow the identification of the genes involved in AR formation in this species, which will contribute to our basic understanding of the molecular events leading to this complex developmental response.

\section{Methods}

\section{Plant material and growth conditions}

Stem cuttings were pinched from several mother plants of the $2003 R 8$ and 2101-02 MFR cultivars by skilled operators at noon on $2^{\text {nd }}$ December $2013(-23$ h). About 500 stem cuttings of each cultivar were wrapped in plastic bags after pinching and were sent refrigerated and in complete darkness to the laboratory $(-15 \mathrm{~h})$. Next, the stem cutting bases were submerged for $15 \mathrm{~h}$ in a $100 \mathrm{ml}$-water solution containing either mock or an auxin cocktail (1.5 $\mu \mathrm{M}$ indole-3-butyric acid [IBA; Duchefa, The Netherlands] and $1 \mu \mathrm{M} \alpha$-naphtalene acetic acid [NAA; Duchefa,
The Netherlands]). After the treatment, the cuttings were individually planted in 70-well trays containing moistened perlite plugs $\left(4.5 \times 4.5 \times 4.5 \mathrm{~cm} ; 90 \mathrm{~cm}^{3}\right)$, and their basal regions were collected at $0,6,24$ and $54 \mathrm{~h}$ after planting (hAP) in a walk-in growth chamber that was set at $22 \pm 2^{\circ} \mathrm{C}, 70 \%$ relative humidity and under continuous fluorescent light with an average photosynthetic photon flux density of $40 \mu \mathrm{mol} \mathrm{m} \mathrm{m}^{-2} \mathrm{~s}^{-1}$. Three biological replicates, each consisting of fifteen stem cutting bases $(\sim 5 \mathrm{~mm}$ long), were collected per cultivar, treatment and time point, and were immediately frozen in liquid $\mathrm{N}_{2}$. To minimize variation due to subtle environmental differences within the growth chamber, an incomplete block experimental design was used. The experimental design used for sample collection is shown in Fig. 1a.

\section{RNA isolation, library construction and Illumina sequencing}

For each sample, total RNA from $\sim 120 \mathrm{mg}$ of powdered stem cutting base tissue that was kept at $-65^{\circ}$ $C$ was extracted using Spectrum $^{\text {TM }}$ Plant Total RNA Kit (Sigma-Aldrich, USA). The RNA integrity was confirmed using the 2100 Bioanalyzer (Agilent Technologies, USA). External RNA Controls Consortium RNA Spike-In mixes (Life technologies, USA) were used to assess the sensitivity and dynamic range of the experiment. The samples were prepared for sequencing using the TruSeq RNA Sample Preparation Kit v2 (Illumina, USA). Illumina 100 bp paired-end sequencing on the HiSeq2000 was carried out by Macrogen, Korea. The raw Illumina reads were preprocessed using our in-house quality control pipeline. The 3'ends with a quality score below 20 were trimmed.

\section{Reference genome: feature re-annotation and functional annotation}

We used the carnation reference genome assembly released by [27]. We extended the available gene prediction using transcript sequence evidence. To this end, we assembled a comprehensive transcriptome with RNA sequencing (RNA-Seq) data comprising the sum of eight different tissues and cultivars. Each of the transcriptome assemblies was done using a genome-guided hybrid approach with Trinity [28]. Next, we leveraged this information by first aligning the transcripts to the annotated genome. The best alignments between transcript and genome annotation (those spanning at least $90 \%$ of the transcript length) were selected and subsequently clustered in groups based on a minimum overlap of $30 \%$ between alignments. These clusters were used as evidence to update the existing feature annotation with PASA [29]. To obtain a functional annotation, 

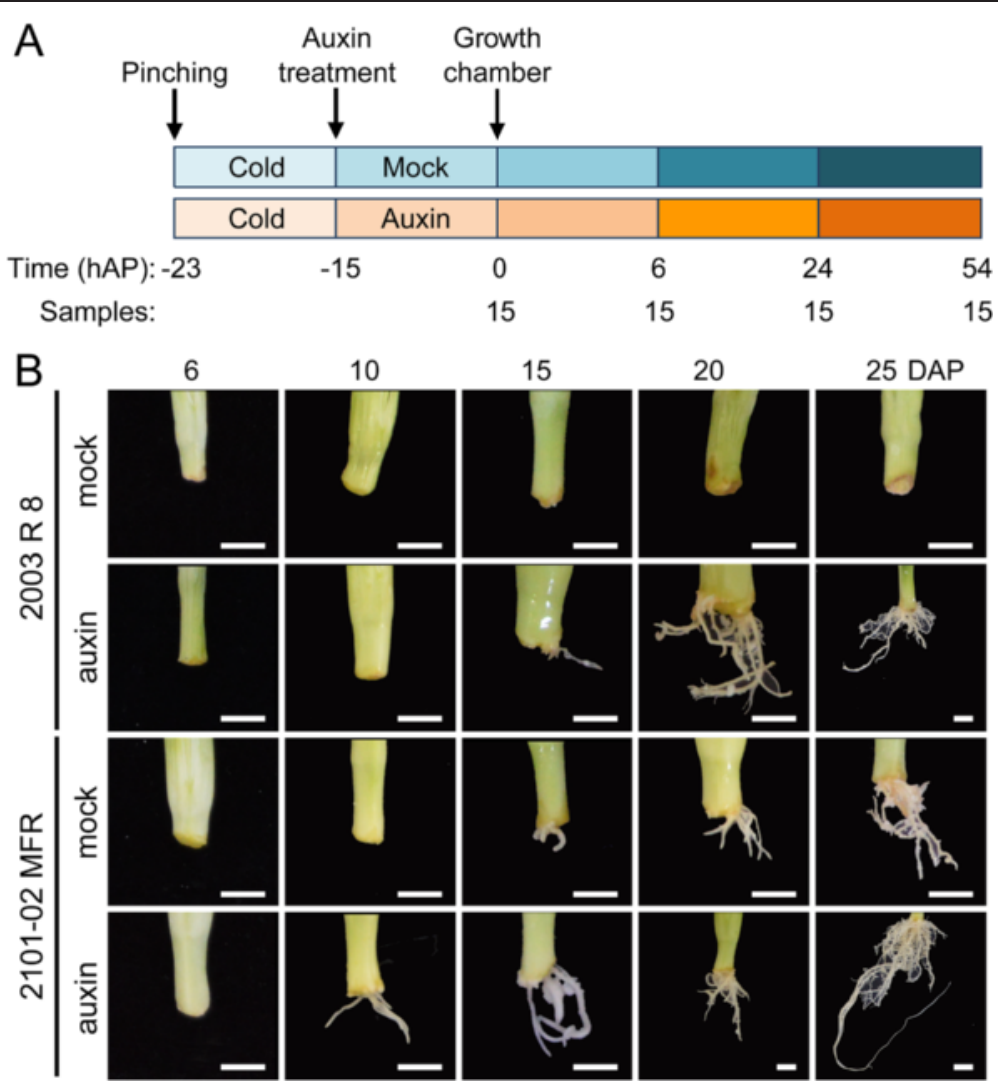

Fig. 1 Experimental design and time-series analysis of AR formation in two carnation cultivars. a Schematic representation of the experimental design followed in our time-course experiment. hAP: hours after planting. $\mathbf{b}$ Representative images of the basal stem of carnation cuttings of the $2003 R 8$ and 2101-02 MFR cultivars between 6 and 25 days after planting (DAP). Scale bars: $5 \mathrm{~mm}$

open reading frames (ORFs) were inferred from the updated, evidence-based gene models using Transdecoder [29]. We then blasted these ORFs against a database comprising all the complete proteins from core-eudicots with a Gene Ontology (GO) [30] annotation (approximately 200,000 proteins). The use of a relatively small and highly informative set of proteins as a database increases power (smaller search-space, smaller e-values) and minimizes the chance for noisy alignments with non-homologous or non-annotated proteins. The ORFs were also compared to model profiles from the Pfam domain database [31] using HMMER [32]. The output from these sequence comparisons was integrated using ARGOT2 [33] to assign one (or several) GO annotation to each ORF. Beside this functional annotation, and in order to include information that is mainly available in model species, we mapped carnation genes to their putative Arabidopsis thaliana (Arabidopsis) orthologues from the The Arabidopsis Information Resource (TAIR) database [34] by means of: i) reciprocal best-hits between carnation and Arabidopsis proteomes, and ii) one-way best-hits $(\mathrm{OBH})$ of carnation to the Arabidopsis proteome.

Exploratory data analysis and differential expression tests Prior to the differential expression analysis with the DESeq2 package [35], we assessed the overall similarity between samples in order to check that it fitted the expectation from the experimental design. We calculated the Euclidean distance between samples using regularized-log transformed expression values to avoid that a few highly variable genes dominated the distance measure. We also used principal-component analysis (PCA) to examine the similarity between samples according to the components that explain most of the variance in the data as shown in Additional file 1: Figure S1.

Using the DESeq2 package we fitted generalized linear models of gene expression. The significance of the coefficient of the fitted models was inferred using a Wald test. To increase power, we filtered out genes with zero counts in all the samples, which reduces the burden of a strong multiple test correction [36]. This reduced the 
data to 37,849 genes. Biological replicates were considered for each time point, as previously described [37]. Functional enrichment was tested with topGO [38] for the lists of resulting differentially expressed genes (DEG).

\section{Gene expression analysis by quantitative reverse-transcription PCR}

The selection of candidate genes for their experimental validation by quantitative reverse-transcription PCR (qRT-PCR) was based on the following criteria: $i$ ) high relative expression level in the RNA-Seq experiment at 0 hAP, ii) the function of its putative Arabidopsis ortholog was related to root growth and development, and iii) a dynamic expression range across the evaluated period. Six genes fulfilling these criteria were chosen for qRT-PCR analysis: Dca5879, Dca23172, Dca29160, Dca30890, Dca40234 and Dca43825. For primer design, small amplicons (90 to $140 \mathrm{bp}$ ) were chosen within the first third of the cDNA sequences. Whenever possible, forward and reverse primers bind to different exons and the reverse primer was designed to hybridize with two consecutive exons to avoid amplification of genomic DNA.

The first strand cDNA was synthesized from $1 \mu \mathrm{g}$ of purified RNA using the iScript Reverse Transcription Supermix for RT-qPCR (Bio-Rad, USA). The resulting cDNA was diluted by adding $40 \mu \mathrm{l}$ of sterile distilled water. Fourteen $\mu \mathrm{l}$ reactions were prepared with $7 \mu \mathrm{l}$ of the SsoAdvanced ${ }^{\mathrm{Tw}}$ Universal SYBR $^{\circ}$ Green Supermix (Bio-Rad), $5 \mu \mathrm{M}$ of specific primer pairs (Additional file 2: Table S1) and $1 \mu \mathrm{l}$ of cDNA. PCR amplifications were carried out in 96-well optical reaction plates on a Step One Plus Real-Time PCR System (Applied Biosystems, USA). Two independent RNA isolates and three technical replicates were used per cultivar, treatment and time point assayed. The thermal cycling program started with a step of $10 \mathrm{~s}$ at $95{ }^{\circ} \mathrm{C}$, followed by 40 cycles $(15 \mathrm{~s}$ at $95{ }^{\circ} \mathrm{C}$ and $60 \mathrm{~s}$ at $60^{\circ} \mathrm{C}$ ), and the melt curve (from $60^{\circ}$ $\mathrm{C}$ to $95^{\circ} \mathrm{C}$, with increments of $0.3^{\circ} \mathrm{C}$ every $5 \mathrm{~s}$ ). Dissociation kinetics and agarose gel loading of the amplified products confirmed their specificity.

Primer pair validation and relative quantification of gene expression levels were performed by using the $2^{-\Delta \Delta C T}$ method [39]. The Dca17200 gene (the putative homolog of the Arabidopsis housekeeping gene ACTIN2; AT3G18780) was chosen for normalization of the assayed genes as its expression was constant among the different cultivars, treatments and time points studied. All samples were compared to the expression level of the control treatment (mock) at the zero-time point $(0 \mathrm{hAP})$. The average of fold-change values were used for graphic representation.

\section{Time-course analysis}

To cluster genes according to their time-course profile, we reformatted cross-sectional data where each sample corresponds to cuttings from different plants (i.e., destructive sampling) as longitudinal data. To this purpose, the normalized counts of replicated samples at different time points were paired, producing complete time courses. To handle the missing data of one of the mock replicates at 0 hAP in the cultivar $2003 R 8$, missing values were imputed averaging the normalized counts from the other two replicates at the same time point and cultivar. Gene clustering and GO enrichment analysis within clusters was performed in STEM [40] using default parameters (STEM Clustering Method). In order to increase the signal-to-noise ratio, we filtered out genes with a $\log$ expression difference across time points smaller than 1.25 and correlation between replicates smaller than 0.75 .

\section{Light microscopy}

For each cultivar and treatment, $\sim 5 \mathrm{~mm}$ long segments from the base of the stem cuttings were sectioned at different time points $(0,6,24$ and $54 \mathrm{hAP})$. Samples were fixed in a FAA/Triton solution ( $1.85 \% \mathrm{v} / \mathrm{v}$ formaldehyde, $45 \%$ ethanol, $5 \%$ acetic acid, and $1 \%$ Triton X-100) for 8 $\mathrm{h}$ on a light vacuum (400 mbar) until the tissue sank. Samples were then kept in the FAA/Triton solution for 3 days at $4{ }^{\circ} \mathrm{C}$. The fixed tissue was rinsed 3 times in $0.1 \mathrm{M}$ sodium phosphate buffer $(\mathrm{pH} 7.2)$ before dehydrating in a graded ethanol series (70, 80, 90 and $96 \%$ ethanol, $60 \mathrm{~min}$ each step). Dehydrated samples were then embedded in Technovit 7100 resin (Heraeus Kulzer GmbH, Germany) according to the manufacturer's instructions with slight modifications, as follows. Samples were immersed in the pre-infiltration solution (50\% v/v resin and $50 \%$ ethanol) for $2.5 \mathrm{~h}$. Then, stem cutting samples remained $4 \mathrm{~h}$ in the infiltration solution on a light vacuum at room temperature and polymerized for $20 \mathrm{~h}$ at $4{ }^{\circ} \mathrm{C}$. Thin sections of $7 \mu \mathrm{m}$-thickness were cut using a tungsten microtom knife (MICROM International GmbH, Germany) on a HS $350 \mathrm{~S}$ rotary microtome (MICROM International GmbH). Sections were stained either with $0.05 \%$ weight/volume $(\mathrm{W} / \mathrm{V})$ toluidine blue (Sigma-Aldrich) or $0.05 \% \mathrm{~W} / \mathrm{V}$ ruthenium red (SigmaAldrich) in water and mounted in Eukitt (Chem-Lab NV, Belgium). Samples were observed using a brightfield Motic BA210 microscope (Motic Spain, Spain) and selected images were captured with a built-in Moticam 580INT documentation station (Motic Spain) and processed with Adobe Photoshop CS3.

\section{Phytohormone extraction and analysis}

Phytohormones were extracted and analysed according to [41]. Briefly, $\sim 100 \mathrm{mg}$ of frozen tissue from the same batches used for the RNA-Seq experiment were extracted 
twice with $1 \mathrm{ml}$ of methanol/water $80 \%$, centrifuged at 20,000 $\mathrm{g}$ for $15 \mathrm{~min}$. at $4{ }^{\circ} \mathrm{C}$, the supernatant was passed through a C18 cartridge, and the samples were collected in a 5-ml tube for speed-Vac evaporation to dryness. The residue was resuspended in $1 \mathrm{ml}$ methanol/water $20 \%$. Ten $\mu \mathrm{l}$ of filtrated extract were injected in a U-HPLC-MS system consisting of an Accela Series U-HPLC (ThermoFisher Scientific, USA) coupled to an Exactive mass spectrometer (ThermoFisher Scientific) using a heated electrospray ionization interface. Mass spectra were obtained using the Xcalibur software version 2.2 (ThermoFisher Scientific). For quantification of the plant hormones, calibration curves were constructed for each analysed component $\left(1,10,50\right.$, and $\left.100 \mu \mathrm{g} \mathrm{l}^{-1}\right)$ and corrected for $10 \mu \mathrm{g} \mathrm{l}^{-1}$ deuterated internal standards. Recovery percentages ranged between 92 and $95 \%$.

\section{Transcription factor analysis}

To find out which transcription factor (TF) families are likely to play a more important role along the experimental process, we analysed their enrichment among genes annotated with the function "sequence-specific DNA binding transcription factor activity" (GO:0003700). In correspondence with the filtering criteria for the time-course analysis, we excluded genes with a log expression difference across time smaller than 1.25 and correlation between replicates smaller than 0.75. Carnation genes with predicted transcription factor activity were classified in families via their putative Arabidopsis orthologs, based on the OBH method (see functional annotation section). The family classification of these orthologs was obtained from the Database of Arabidopsis Transcription Factors [42]. Genes mapped to TF families were further categorised as upregulated or downregulated according to their profiles in the timecourse analysis at $54 \mathrm{hAP}$. For each category, a Fisher exact test was done to assess significant enrichment. P-values were adjusted for multiple testing (Benjamini-Hochberg).

\section{Results}

\section{Sequencing and transcriptome assembly supports the discovery of novel genes expressed in the stem cutting base}

In a recent study [26] we characterized AR formation in a collection of 10 carnation cultivars. The $2003 R 8$ and the 2101-02 MFR cultivars have been chosen for further studies due to their differences in rooting performance and in their differential response to a mild auxin treatment during rooting (Fig. 1b). The bad-rooting behaviour of the $2003 R 8$ cultivar, which was mostly caused by a delay in AR initiation, was partially restored by exogenous auxin application.

Several cDNA libraries prepared from stem cutting bases of mock-treated and auxin-treated samples at particular time points during adventitious rooting $(0,6$,
24 and 54 hAP) were sequenced (see Methods; Fig. 1a). As a result, 3,683 million of raw reads were obtained. The amount of expression data generated in our study had the potential to transform the boundaries and extent of previous feature annotations in the carnation genome [27]. Genome-guided assemblies were performed with the purpose of serving as sequence evidence for a genome reannotation. Thus, our updated, evidence-based annotation comprised 59,396 transcripts, corresponding to 57,641 genes, with an average length of 2,856 bp (Table 1). We were able to merge exons from genes that had been previously predicted as separate coding sequences [27]; these merges resulted in a more complete or contiguous annotation for 394 genes and their corresponding transcripts (see Additional file 3). In order to quantify the improvements of the new annotation in relation to the former annotation, we compared the proportion of reads mapping to each of them and also generated a number of descriptive statistics (Table 1).

\section{Time-dependent comparison of the auxin treatment identifies 1,286 differential expressed genes (DEGs) in response to the auxin stimulus}

We then tested the effects in gene expression of factors like cultivar, treatment and time point using different models and contrasts (Table 2). Three questions were addressed: $i$ ) for which genes does the cultivar factor have a significant effect? (Table 2, test 1), ii) which genes change their expression over each pair of time points? (while accounting for cultivar-specific effects; Table 2, test 2-13) and iii) how does the auxin treatment affect gene expression distinctively at each time point for each cultivar? (Table 2, test 14-21). To reduce the complexity of the model, in this last case, we made subsets of samples belonging to each cultivar and estimated the parameters separately.

In all cases, expression models were fitted to our timecourse study by treating each time point as a different "experimental group", even though the inherent ordering and spacing provided by time points is ignored then.

To investigate the dependencies between treatments and time, explicitly addressing the question of when a gene is differentially expressed, we modeled the interaction between time and treatment as a covariate. Of 57,641 genes, we filtered out genes that were not

Table 1 Comparison between the previously published annotation and the updated genome annotations

\begin{tabular}{lll}
\hline Feature & Yagi et al. [27] & Evidence-based annotation \\
\hline Gene count & 56,137 & 57,641 \\
Transcript count & 56,382 & 59,396 \\
Average transcript length & 2,742 & 2,856 \\
Median transcript length & 2,065 & 2,125 \\
\hline
\end{tabular}


Table 2 Differential expression tests

\begin{tabular}{|c|c|c|c|c|}
\hline Test & Data subset & Model formula & Contrast tested & DEGs \\
\hline 1 & All & $\mathrm{C}+\mathrm{Ti}+\mathrm{C}: \mathrm{Ti}$ & 2003 R 8 vs. 2101-02 MFR & 23,029 \\
\hline 2 & All & $C+T i+C: T i$ & 2003 R 8:Ti 1 vs.2003 R 8:Ti 2 & 3,820 \\
\hline 3 & All & $\mathrm{C}+\mathrm{Ti}+\mathrm{C}: \mathrm{Ti}$ & 2003 R 8:Ti 1 vs. 2003 R 8:Ti 3 & 4,645 \\
\hline 4 & All & $C+T i+C: T i$ & 2003 R 8:Ti 1 vs. 2003 R 8:Ti 4 & 5,880 \\
\hline 5 & All & $C+T i+C: T i$ & 2003 R 8:Ti 2 vs. 2003 R 8:Ti 3 & 2,828 \\
\hline 6 & All & $\mathrm{C}+\mathrm{Ti}+\mathrm{C}: \mathrm{Ti}$ & 2003 R 8:Ti 2 vs. 2003 R 8:Ti 4 & 2,983 \\
\hline 7 & All & $\mathrm{C}+\mathrm{Ti}+\mathrm{C}: \mathrm{Ti}$ & 2003 R 8:Ti 3 vs. 2003 R 8:Ti 4 & 690 \\
\hline 8 & All & $C+T i+C: T i$ & 2101-02 MFR:Ti 1 vs. 2101-02 MFR:Ti 2 & 11,536 \\
\hline 9 & All & $C+T i+C: T i$ & 2101-02 MFR:Ti 1 vs. 2101-02 MFR:Ti 3 & 12,694 \\
\hline 10 & All & $C+T i+C: T i$ & 2101-02 MFR:Ti 1 vs. 2101-02 MFR:Ti 4 & 12,129 \\
\hline 11 & All & $\mathrm{C}+\mathrm{Ti}+\mathrm{C}: \mathrm{Ti}$ & 2101-02 MFR:Ti 2 vs. 2101-02 MFR:Ti 3 & 9,336 \\
\hline 12 & All & $C+T i+C: T i$ & 2101-02 MFR:Ti 2 vs. 2101-02 MFR:Ti 4 & 9,125 \\
\hline 13 & All & $C+T i+C: T i$ & 2101-02 MFR:Ti 3 vs. 2101-02 MFR:Ti 4 & 3,430 \\
\hline 14 & 2003 R 8 & $\mathrm{Ti}+\mathrm{Ti}: \mathrm{Tr}$ & Ti 1:Aux vs. Ti 1:Mock & 86 \\
\hline 15 & 2003 R 8 & $\mathrm{Ti}+\mathrm{Ti}: \mathrm{Tr}$ & Ti 2:Aux vs. Ti 2:Mock & 1 \\
\hline 16 & 2003 R 8 & $\mathrm{Ti}+\mathrm{Ti}: \mathrm{Tr}$ & Ti 3:Aux vs. Ti 3:Mock & \\
\hline 17 & 2003 R 8 & $\mathrm{Ti}+\mathrm{Ti}: \mathrm{Tr}$ & Ti 4:Aux vs. Ti 4:Mock & 6 \\
\hline 18 & 2101-02 MFR & $\mathrm{Ti}+\mathrm{Ti}: \mathrm{Tr}$ & Ti 1:Aux vs. Ti 1:Mock & 1,188 \\
\hline 19 & 2101-02 MFR & $\mathrm{Ti}+\mathrm{Ti}: \mathrm{Tr}$ & Ti 2:Aux vs. Ti 2:Mock & 21 \\
\hline 20 & 2101-02 MFR & $\mathrm{Ti}+\mathrm{Ti}: \mathrm{Tr}$ & Ti 3:Aux vs. Ti 3:Mock & 1 \\
\hline 21 & 2101-02 MFR & $\mathrm{Ti}+\mathrm{Ti}: \mathrm{Tr}$ & Ti 4:Aux vs. Ti 4:Mock & \\
\hline
\end{tabular}

Datasets were fitted to the corresponding formula ( $C=$ cultivar, $\mathrm{Ti}=$ time point, $\mathrm{Tr}=$ treatment; additive effects are represented by "+"; ":" represents interaction). After model fitting, selected factors were tested (Ti $1=0$ hAP, Ti $2=6$ hAP, Ti $3=24$ hAP, Ti $4=54$ hAP). DEG: Differential expressed genes; $P$-adj. $<0.05$ (Benjamini-Hochberg correction)

expressed (0 counts), resulting in a total of 37,936 genes tested for the subset of cultivar 2101-02 MFR and 37,849 for the cultivar $2003 R 8$ subset. The factorial analysis identified a total of 1,286 distinct genes as differentially expressed between auxin-induced and control cuttings over different time points (Table 2, test 14-21). Most auxin-related expression changes took place in the initial time points (0 hAP vs. $6 \mathrm{hAP}$ ). Among them, DEGs of 2101-02 MFR were associated (Fisher exact test) to functions like photosynthesis (GO:0015979; $P<0.001)$ and chlorophyll binding (GO:0016168; $P<0.001$ ). As for the same comparison in the cultivar with poor rooting performance, $2003 R 8$, the functions associated to DEG were translational initiation factor activity (GO:0003743, $P=0.0025)$, and negative regulation of signal transduction (GO:0009968, $P=0.0024$ ), among others.

\section{Clustering of time-course expression profiles reveals co-expression of functionally related genes}

To get some insight into the specific pathways regulated at different time points during AR formation in the two cultivars studied, we performed a GO-enrichment analysis for the sets of DEGs shown in Fig. 2a. In the $2003 R 8$ cultivar, the GO categories "protein amino acid phosphorylation"
(GO:0006468; 105 genes; $P<0.001)$ and "transmembrane transport" (GO:0055085; 50 genes; $P<0.001$ ) were specifically and significantly enriched at 6-24 and 24-54 hAP, respectively. Interestingly, the "auxin-activated signalling pathway" (GO:0009734; 11 genes; $P<0.001$ ) category was found significantly enriched among DEGs shared between $0-24$ hAP in this cultivar. Conversely, in the 2101-02 MFR cultivar, the GO category "hormone-mediated signalling pathway" (GO:0009755; 58 genes; $P<0.001$ ) was specifically enriched at $0-6$ hAP. Moreover, the "auxin-activated signalling pathway" (GO:0009734; 34 genes; $P<0.001$ ) and "cell cycle" (GO:0007049; 79 genes; $P<0.001$ ) categories were found significantly enriched for DEGs shared between 0 and 24 hAP. The GO categories "glucose catabolic process" (GO: 0006007; 41 genes; $P<0.001$ ), and "cellulose biosynthetic process" (GO:0030244; 22 genes; $P=0.002$ ) were significantly enriched specifically at 6-24 hAP, whereas at 24-54 hAP the most significant GO-enrichment was found for genes assigned to the "response to stress" (GO:0006950; 46 genes; $P=0.007$ ) category.

To obtain a more general view of the functions involved in the early stages of AR formation, we transformed the GO functional annotation into its cut-down 


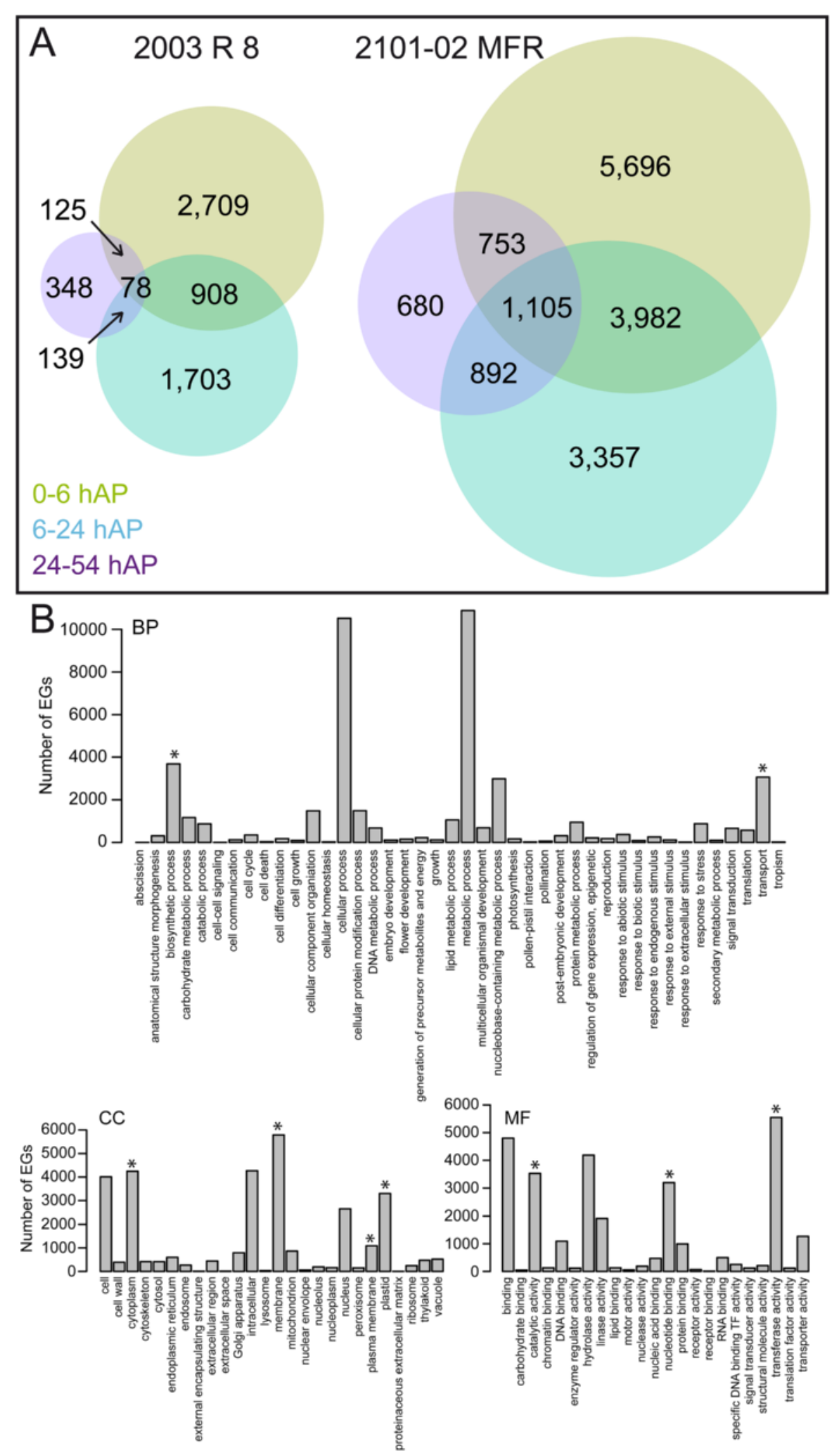

Fig. 2 (See legend on next page.) 
(See figure on previous page.)

Fig. 2 Differentially expressed genes (DEGs) over time during AR formation. a Venn diagram illustrating DEGs ( $P<0.05$, Benjamini-Hochberg correction) in the stem cutting base of the 2003 R 8 and 2101-02 MFR cultivars over time. $\mathbf{b}$ Gene ontology (GO) classification of genes whose expression changes more than 1.25 log-fold over the time-course with respect to biological process (BP), cellular component (CC) and molecular function (MF). Asterisks indicate significant enrichment of genes $(P<0.05)$

version (Plant GO slim) for the 14,554 DEGs in the 2101-02 MFR cultivar between 0 hAP and 6 hAP (Fig. 2b). The Biological Process (BP) classification of DEGs highlighted a significant enrichment $(P<0.001)$ for the following GO categories: "biosynthetic process" (GO:0009058) and "transport" (GO:0006810). Among the Cellular Component (CC) categories, "plastid" (GO:0009536) and "plasma membrane" (GO:0005886) were the most significantly enriched ones. In terms of Molecular Function (MF), a significant enrichment was found for genes at the categories "transferase activity" (GO:0016740) and "nucleotide binding" (GO:0000166). These results indicated that large expression changes are taking place at transcriptome level in the stem cutting base during the initial stages of AR formation in this cultivar.

\section{Validation of expression of some of the genes detected during AR formation}

The reliability of our transcriptome profiling dataset was validated by examining the expression of selected genes by using qRT-PCR and by comparing them to the normalized data obtained in the RNA-Seq analysis (see Methods). We found highly significant and positive correlations between qRT-PCR and RNA-Seq results for both cultivars in all time points and treatments (Fig. 3a-b). Additional statistical analysis revealed that the variation observed between qRT-PCR and RNA-Seq results depended largely on the expression levels of the studied genes (Additional file 4: Figure S2). Thus, for genes with very low or very high numbers of RNA-Seq reads, the qRT-PCR validation was less accurate. Representative examples of the results obtained for genes with contrasting expression profiles are shown in Fig. 3c-f. While the expression levels of Dca5879 were only varying over time (Fig. 3c-d), those of Dca29160 were also depending on cultivar and the auxin treatment (Fig. 3e-f).

\section{Gene set enrichment analysis in the $2003 R 8$ cultivar}

As the number of DEGs between auxin-treated and mocktreated samples in the $2003 R 8$ cultivar was scarce (see above), we did not distinguish between treatments in this cultivar when performing a GO-enrichment analysis using STEM (see Methods). The expression of 7,341 genes was found to be specifically regulated during AR formation in the $2003 R 8$ cultivar and 4,599 of these genes were clustered along different expression profiles and further classified into four major groups based on their expression pattern between 0-6 hAP and 6-54 hAP: DownDown (DD), DownUp (DU), UpDown (UD), and UpUp (UU) (Fig. 4a).

1,286 genes were gradually repressed (DD group) during AR formation in these conditions. Some of the most significantly-enriched GO categories within this group were "response to auxin" (GO:0009733; 15 genes; $P<$ 0.001 ) and "ion transport" (GO:0006811; 46 genes; $P<$ 0.001). We assigned 1,381 additional genes to the DU group. About two-thirds of these genes showed an early repression and later became upregulated above their expression at 0 hAP (labelled as "a" in Fig. 4a). The remaining genes in this group, which were quickly downregulated and whose levels were more-or-less restored to initial levels at later time points (labelled as "b" in Fig. 4a), showed enrichment in "photosynthetic membrane" (GO:0034357; 30 genes; $P<0.001)$ encoding genes. Some of the most significantly-enriched GO categories within the DU group as a whole were "cell wall organization or biogenesis" (GO:0071554; 51 genes; $P<0.001)$, “cytoskeleton" (GO:0005856; 46 genes; $P<0.001$ ), and "cellular carbohydrate metabolic process” (GO:0044262; 63 genes; $P<$ 0.001). Another 790 genes showing a biphasic response were classified into the UD group. Finally, 1,142 genes within the UU group were ranked for GO enrichment: "cell division" (GO:0051301; 32 genes; $P<0.001)$, "microtubule" (GO:0005874; 52 genes; $P<0.001$ ), and "cell wall organization or biogenesis" (28 genes; $P<0.001)$ among others. Interestingly, the expression of most genes included within the "cell division" and "cell wall organization or biogenesis" categories peaked after 6 hAP in agreement with the timing of cell cycle reactivation in the cambium observed for this cultivar, as it is shown later.

\section{Expression profiling in the 2101-02 MFR cultivar and in the response to auxin}

12,525 genes were identified in the 2101-02 MFR cultivar as being specifically expressed during AR formation without exogenous auxin treatment (Fig. 4b). 3,586 genes were assigned to the DD group where one of the most significantly-enriched GO categories was "protein serine/threonine kinase activity" (GO:0004674; 175 genes $[13.8 \%$ of the protein serine/threonine kinase encoding genes with dynamic expression profiles (EGs)]; $P<0.001$ ). 1,737 genes showing a biphasic response were classified into the DU group. Those upregulated at later time points (labelled as "a" in 


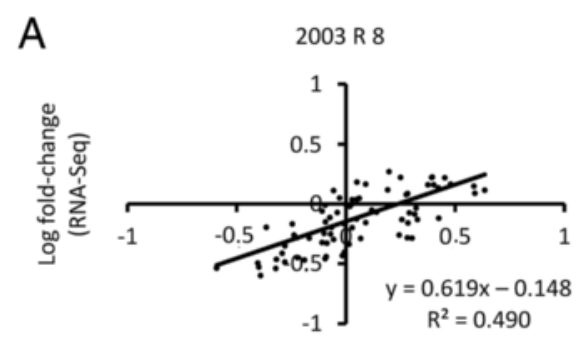

Log fold-change (qRT-PCR)
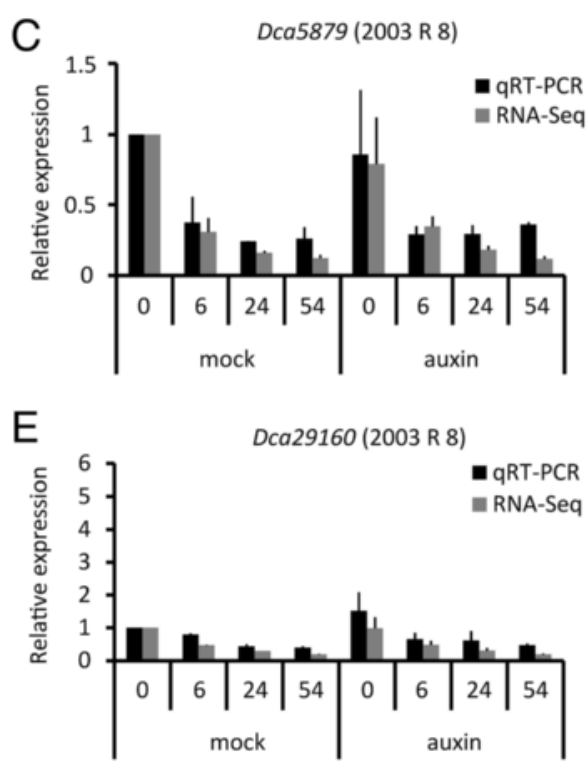

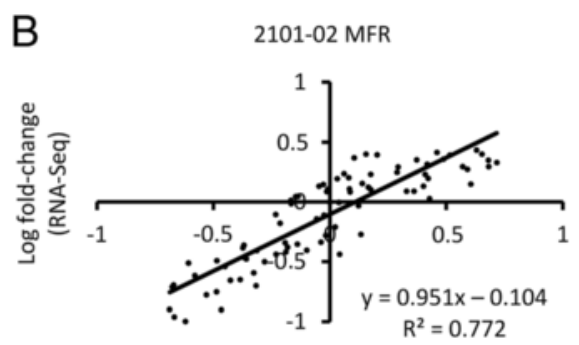

Log fold-change (qRT-PCR)
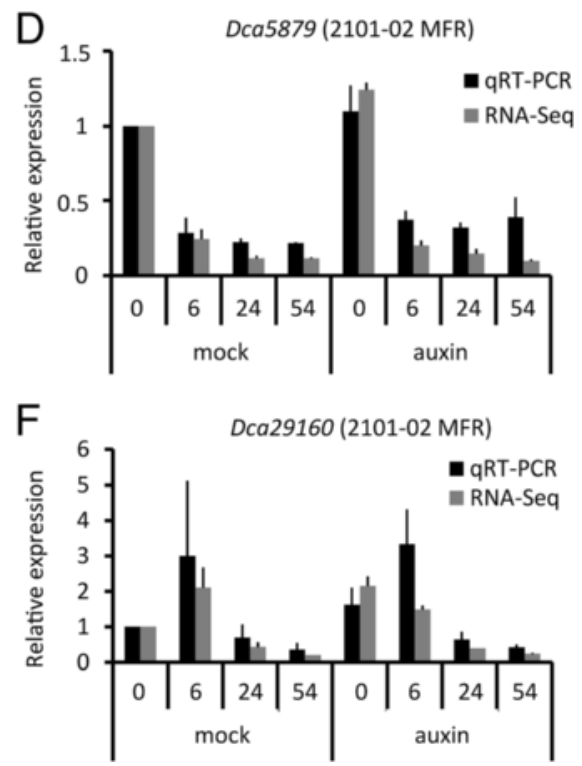

Fig. 3 qRT-PCR validation of RNA-Seq results. $\mathbf{a}$-b Correlation analysis of qRT-PCR and RNA-Seq data from selected genes in the 2003 R 8 (a) and 2101-02 MFR (b) cultivars. Each dot represents the relative expression data for a given gene and a given sample. c-f Bars represent the mean of the relative expression level of qRT-PCR (black) or RNA-Seq (grey) data relative to mock-treated samples at 0 hAP. Error bars indicate the standard deviation for the mean data shown

Fig. 4b) encoded proteins enriched in "cytoskeleton" (GO:0005856; 41 genes; $P<0.001$ ) and "cell division" (17 genes; $P<0.001$ ). Similarly to that found previously for the $2003 R 8$ cultivar, the "photosynthetic membrane" (35 genes; $P<0.001$ ) category was found enriched among genes whose expression levels were restored to basal levels (labelled as "b" in Fig. 4b). Among the biphasic genes that were assigned to the UD group $(1,385)$, one of the significantly-enriched GO categories found was "carbohydrate derivative metabolic process" (GO:1901135; 52 genes; $P=0.002)$. Finally, 2,103 genes were included within the UU group, where the most significantly-enriched GO categories were "cellular carbohydrate metabolic process" (69 genes; $P<0.001)$ and "cell wall organization or biogenesis" (54 genes; $P<0.001$ ). In addition, we found specific GO-enriched categories in profile P29 (Fig. 4b). On the one hand, enriched genes upregulated after 6
hAP (P29) encoded proteins related to "microtubule" (38 genes; $P<0.001)$, "cell division" (24 genes; $P<0.001$ ), and "regulation of cell cycle" (GO:0051726; 23 genes; $P<0.001)$. On the other hand, genes encoding putative chromatin-related functions such as "histone $\mathrm{H} 3$ lysine 9 methylation" (GO:0051567; 14 genes; $P<0.001$ ), or "DNA packaging" (GO:0006323; 14 genes; $P<0.001$ ) were also found significantly enriched.

Additionally, the expression of 9,645 genes was found specifically altered during AR formation in the 2101-02 $M F R$ cultivar after exogenous auxin treatment and 5,568 of these genes were significantly clustered to different expression profiles and grouped as described above (data not shown). We found a substantial overlap between EGs of auxin-treated and mock-treated samples $(61.2 \%$ for the auxin-treated EGs and $79.5 \%$ for the mocktreated EGs). Consistently, no significant differences in the overall trends of EGs were found between auxin- 


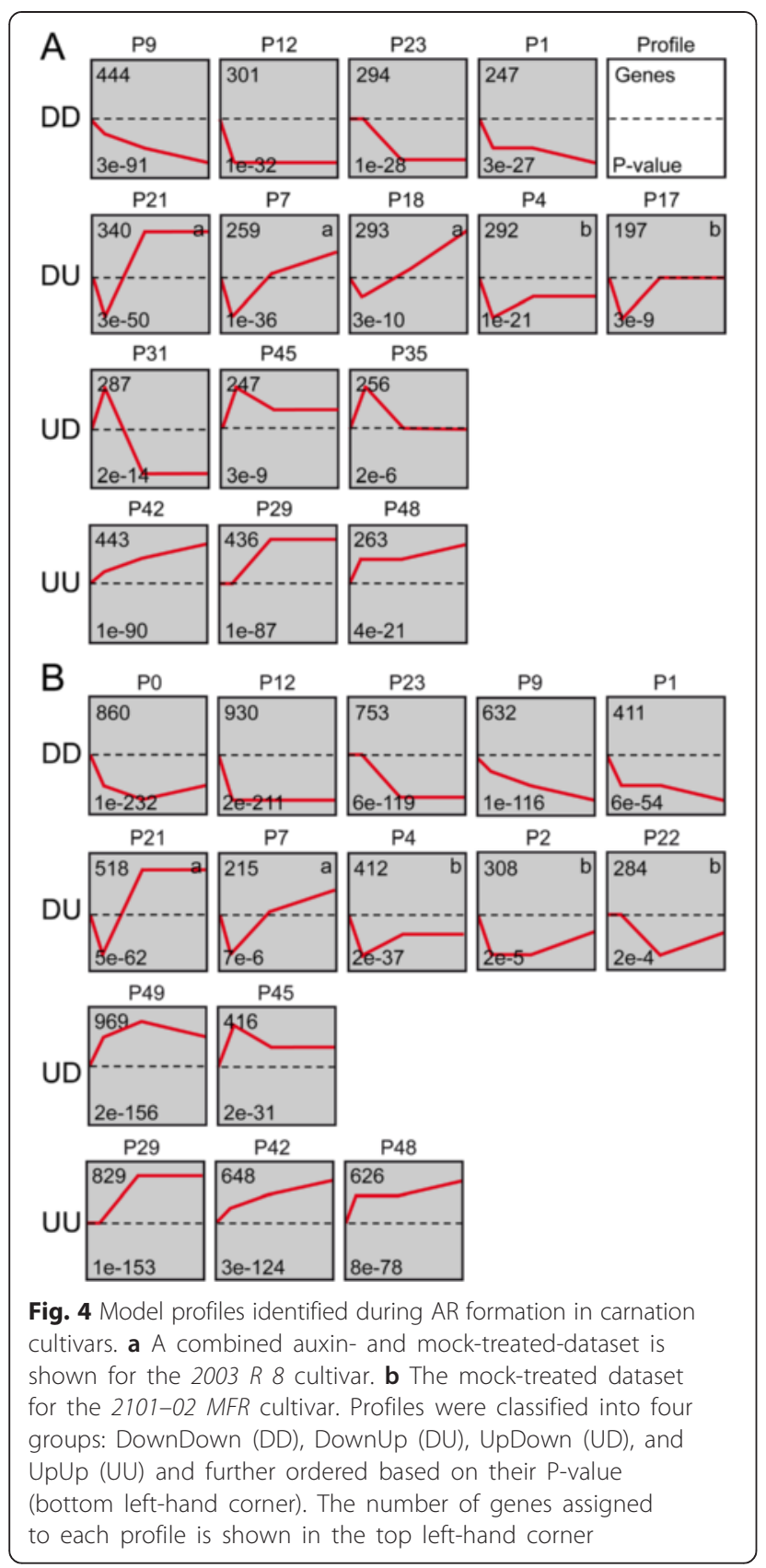

and mock-treated samples for the 2101-02 MFR cultivar (Additional file 5: Figure S3). However, for a small number of genes assigned to specific profiles in mocktreated samples, we found some changes in their expression profiles after the auxin treatment. About half of the EGs-encoding proteins belonging to "microtubule", "cell division" and "histone H3 lysine 9 methylation" were reciprocally assigned to profiles P29 (UU) or P21 (DU) in mock-treated and in auxintreated samples, respectively. We also found that the expression levels of most genes assigned to the "cellular carbohydrate metabolic process" category were complementary at earlier time points in auxin-treated $v s$. mock treated samples.

\section{Comparative transcriptome profiling of AR formation between carnation cultivars for selected GO categories} To identify genes whose expression correlates with the different stages of AR formation and that could be used as markers, we selected EGs belonging to the GO categories "cell division" and "response to auxin" from the different samples studied (see above). We then built heat map representations from log expression data for all these genes (Fig. 5). On the one hand, the expression of some genes encoding proteins related to cell division were clustered together along the time point series with higher expression at earlier time points, independently of cultivar and treatment (Fig. 5a). Interestingly, genes encoding mitotic cyclins (A-type and B-type) showed a peak of expression between 24 hAP and 54 hAP (Fig. 5a), which is in light with the cellular changes observed in the stem cutting base during AR rooting (see next section). Noteworthy, the majority of these genes moderately respond to the auxin treatment by increasing their expression levels at earlier time points (Fig. 5a). On the other hand, a small number of genes displayed contrasting expression profiles between cultivars. Examples for the latter are the Dca37619 and Dca642 genes, which are respectively upregulated and downregulated in $2003 R 8$ compared to the 2101-02 MFR cultivar (Fig. 5a).

Considering the expression profiles of genes assigned to the "response to auxin" category, the effect of the auxin treatment was quite small irrespective of the cultivar, and was mainly restricted to earlier time points (Fig. 5b). However, we found striking differences in the expression of a few of these genes between cultivars, such as Dca1208 and Dca39239, which makes them candidates for further studies to analyze their role in the differential response in auxin-mediated AR initiation between these two cultivars.

\section{Cellular changes in the stem cutting base during AR formation reflects the effect of the auxin signal}

In a previous study we found that cell divisions within the cambial region of the stem cutting base took place between $12 \mathrm{hAP}$ and $24 \mathrm{hAP}$ in a good-rooting cultivar used as a reference [43]. We next characterized the cellular changes occurring within the cambium region in the stem cutting base of the $2003 R 8$ and 2101-02 MFR cultivars both in mock- and auxin-treated samples to understand the differential responses observed in these two cultivars during AR formation. Although the cambial ring of the $2003 R 8$ cultivar displayed a very organized cellular pattern at $0 \mathrm{hAP}$, we found that some regions within the cambium displayed subtle tissue disorganization (Fig. 6a and Additional file 6: Figure S4). Interestingly, we observed an 


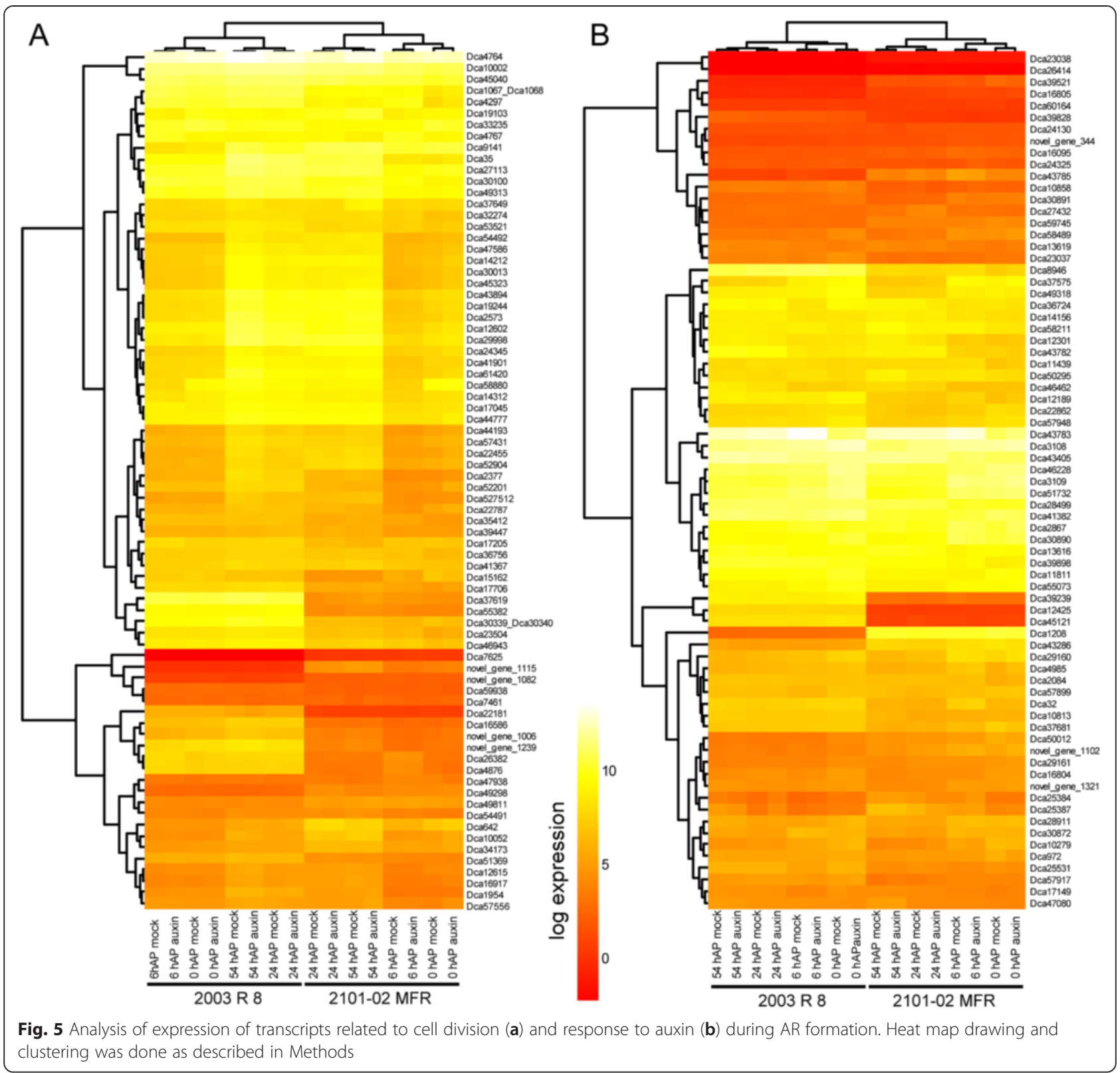

increase in the number of disorganized regions within the cambial ring at later time points, which could reflect local activation of cell divisions (Fig. 6b). In addition, stem cutting bases of the $2003 R 8$ cultivar treated with auxin contained an increased number of these disorganized regions already at 0 hAP (Fig. 6c and 6d). In the 2101-02 MFR cultivar, we observed a higher frequency of small cell clusters within the cambial ring at $0 \mathrm{hAP}$, which at later time points developed as large clusters of meristematic cells with a disorganized internal structure (Fig. 6f). In most cases, these cell clusters appeared juxtaposed but physically isolated by collapsed neighbouring cells (arrowheads in Fig. 6f). In auxin-treated samples however, cell clusters became apparent already at 0 hAP (Fig. 6g), which is indicative of an early activation of cell division in the 2101-02 MFR cultivar. At 54 hAP, cell clusters were clearly evident and their numbers were higher than in mock-treated samples (Fig. 6h).

To confirm our observations, we estimated some cellular parameters in the two contrasting regions identified within the cambium (Additional file 6: Figure S4; see Methods). In the $2003 R 8$ cultivar we observed that the cell division rates significantly differed between organized and disorganized regions at the different time points studied, which seemed not to be affected by the auxin treatment (Additional file 6: Figure S4B). These results suggested that auxin act as a trigger for cell division within a certain population of responsive cambial cells. On the 


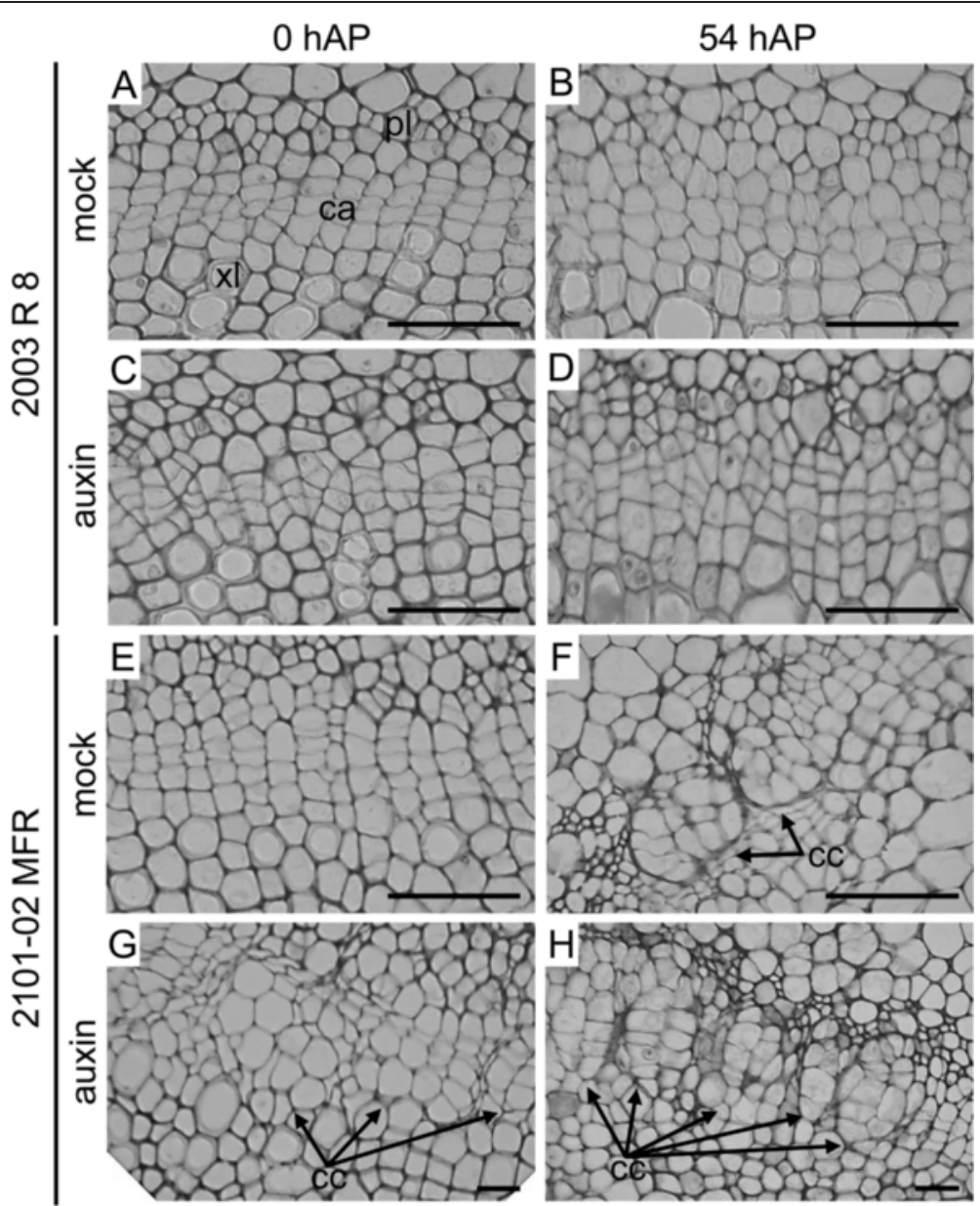

Fig. 6 Morphological changes in the ultrastructure of the basal stem of carnation cuttings during adventitious rooting. Light micrographs were taken from cross-sections of stem cutting basal regions in the 2003 R 8 (a-d) and 2101-02 MFR (e-h) cultivars, either treated with mock (a-b, e-f) or with auxin (c-d, g-h) at different time intervals (0 and 54 hAP). ca, cambium; cc, cell clusters; pl, phloem; xl, xylem. Scale bars: 50 m

other hand, we observed a significant increase in the number of cells within the cambium at later time points for the 2101-02 MFR cultivar, both in organized and disorganized regions (Additional file 6: Figure S4C), which is indicative of a broad activation of cell division within the cambium, as has been previously described for a good-rooting reference cultivar [43]. Interestingly, the division rate at a given time point was found unchanged irrespectively of the auxin treatment (Additional file 6: Figure S4B). Taken together, these results suggested that auxin acts by promoting divisions of quiescent cambial cells rather than by increasing the number of divisions of already dividing cells, the former producing a net increase in the number of cell clusters within the cambial ring.

\section{Morphogenetic hormone levels in the stem cutting base} during AR formation are correlated with rooting performance Several plant hormones play a crucial role in controlling AR formation, with auxin and cytokinin playing opposite roles $[1,44]$. In addition, wounding stimulates ethylene biosynthesis which is known to positively influence AR formation in some species $[45,46]$. We found high levels of endogenous indole-3-acetic acid (IAA) only in the 2101-02 MFR cultivar at 0 hAP, which were quickly downregulated to basal levels (Fig. 7), as previously described [43]. In addition, we found very low levels of trans-zeatin (tZ) in the stem cutting base of the 2101-02 MFR cultivar. In contrast, in the $2003 \mathrm{R} 8$ cultivar, tZ levels steadily increased during the time-course experiment (Fig. 7). Hence, the endogenous auxin/cytokinin ratio estimated as the proportion between IAA and $\mathrm{tZ}$ levels was much higher in the 2101-02 MFR cultivar than in the $2003 R 8$ cultivar for all time points, with the highest ratio found at 0 hAP. In addition, the levels of the 1-aminocyclopropane-1-carboxylic acid (ACC) ethylene precursor were higher in the 2101-02 MFR cultivar than in the $2003 R 8$ cultivar (Fig. 7), which might reflect higher endogenous ethylene production in the $2003 R 8$ cultivar. 

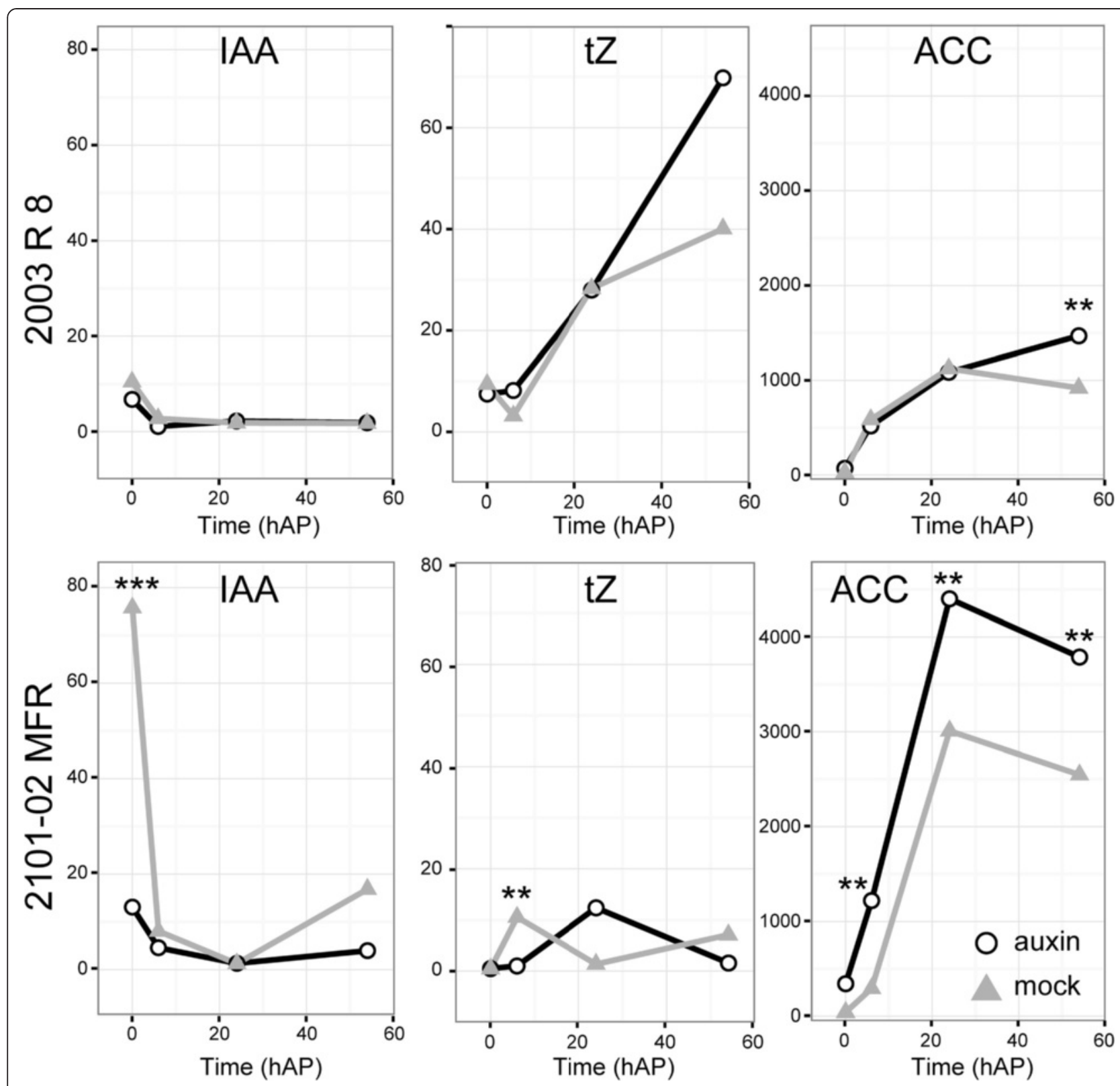

Fig. 7 Changes in the concentration of some phytohormones in the basal stem of carnation cuttings during adventitious rooting. Data for indole-3-acetic acid (IAA), trans-zeatin (tZ) and the ethylene precursor ACC are shown in $\mathrm{ng} / \mathrm{g}$ fresh weight. Time refers to hours after planting (0 hAP). Asterisks indicate significant differences $(P<0.05)$ between treatments for a given sampling time

\section{TF analysis}

By modulating gene transcription at specific times and during specific processes, TFs and their regulatory networks have important roles in development and stress response. A number of transcripts that show significant expression changes during the time-course experiment performed with STEM, encoded putative transcription factors that belonged to 19 transcription factor families (http://planttfdb.cbi.pku.edu.cn/; Fig. 8). To identify the transcription factors that might regulate the differential rooting responses observed between 2101-02 MFR and
$2003 R 8$ cultivars, we performed an enrichment analysis using Fisher's T-test (see Methods). In the $2003 R$ 8 cultivar, a number of genes encoding C2C2-GATA transcription factors, such as Dca7186 and Dca56796, were significantly enriched and showed a clear downregulation of their expression over time (Fig. 8). The WRKY transcription factor family was among the most highly downregulated transcription factor genes irrespective of cultivar and treatment (Fig. 8). Of the 14 carnation genes encoding putative WRKY proteins that were shared between cultivars and treatments, 10 were 


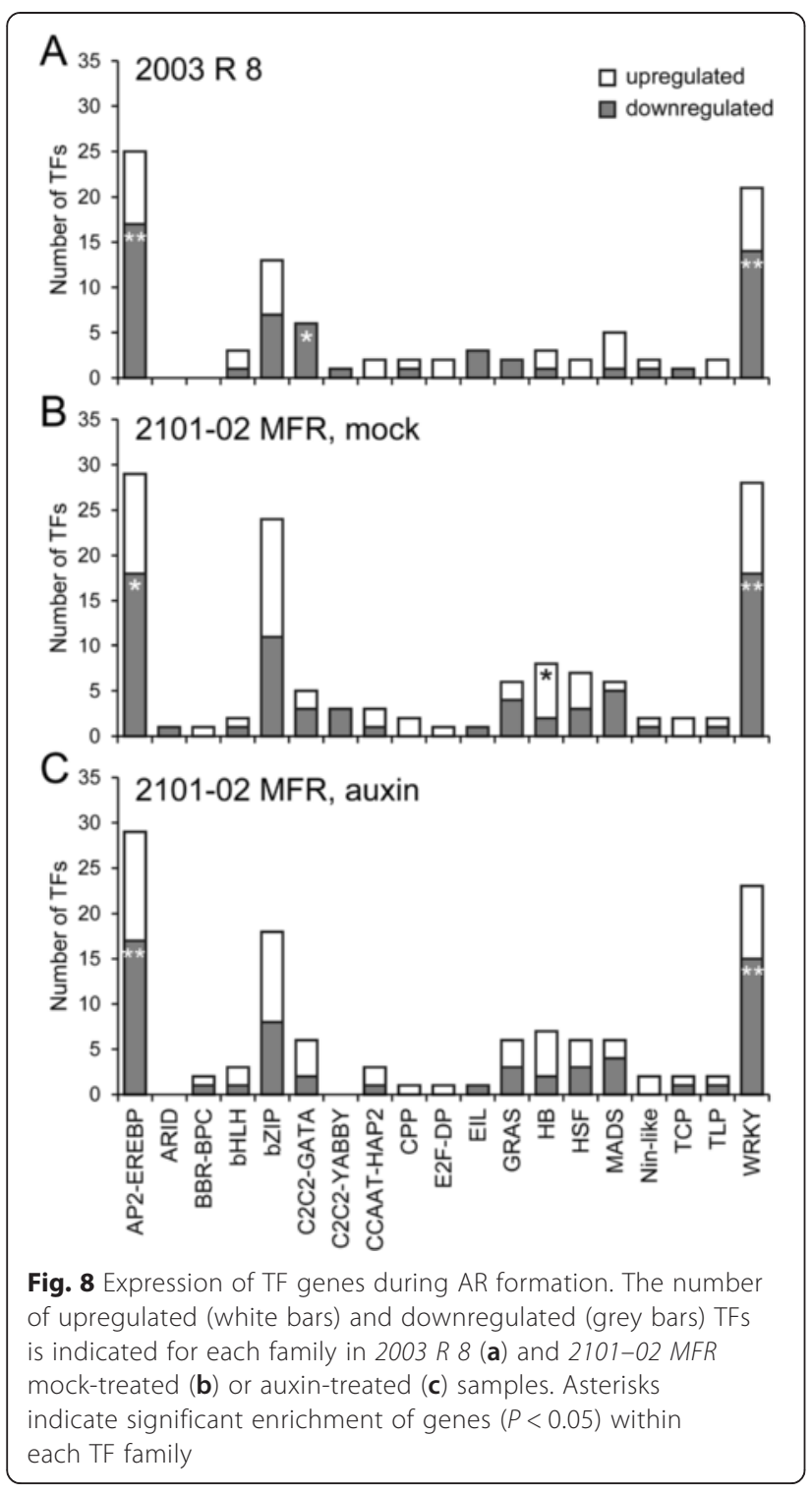

downregulated over the time-course experiment. Interestingly, the expression of Dca28099, the putative carnation ortholog of the Arabidopsis PLETHORA5 gene [47] was found upregulated only in the 2101-02 MFR cultivar after auxin treatment, which emphasizes its value as an early marker for adventitious root formation in this species.

\section{Discussion}

We followed a next-generation sequencing approach to characterize the gene expression profiles in the stem base of two cultivars with contrasting efficiencies of AR formation and in response to exogenous auxin treatment. It was found that the most significant expression differences were driven by the cultivar, less by the time after planting, and the least by the auxin treatment.
Whereas ARs arise directly from cambial tissues in easyto-root species such as poplar, callus formation precedes AR initiation in difficult-to-root species such as Pinus spp. or Eucalyptus grandis [23, 48]. Our histological analysis during root-primordia initiation in two carnation cultivars confirmed that some cambial cells located between the phloem and xylem activate formative (periclinal) divisions in response to the endogenous auxin signal. Next, several clusters of meristematic cells arise along the cambial ring which will later give rise to organized root primordia, as it has been shown previously in the Master reference cultivar [43]. The differences in the rooting ability of the 2003 $R 8$ and 2101-02 MFR cultivars are due to a delay in the early activation of cell divisions in the former. Exogenous auxin treatment had a similar effect on both cultivars: it accelerated the activation of cell division and it caused a higher number of initials within the cambium. As a result, the rate and the number of ARs increased by the auxin treatment in both cultivars [26], which is in agreement with the inductive effect across plant species of exogenously applied auxins [4]. In addition, the analysis of morphogenetic hormone levels in the two carnation cultivars studied indicated that the bad-rooting behavior of $2003 R$ 8 was directly correlated with the low ratio of auxin $v s$. cytokinin levels found in this cultivar.

Consistent with transcriptome data from other species [13, 46], several genes encoding known regulators of auxin response were found differentially regulated during the early stages of AR formation ( 0 hAP and $6 \mathrm{hAP})$ in both cultivars. Several EGs encoding putative Aux/IAA corepressors showed specific upregulation 6 hAP in the 2101-02 MFR cultivar and they were found to be transiently induced by the auxin treatment in both cultivars (Fig. 5b). Among them were Dca28911, Dca30890 and Dca58489, putative homologs of SHY2 (also known as IAA3) [49], MASSUGU2 (IAA19) [50] and SOLITARY ROOT (IAA14) [51]. In addition, some other genes encoding Aux/IAA proteins, such as Dca39239 and Dca39521, showed divergent expression levels in the two cultivars studied (Fig. 5b), which suggested that a differential auxin response could explain the differences between good-rooting and bad-rooting cultivars in this species. However, as both cultivars are able to initiate AR formation in response to exogenous auxin, we believe that the auxin response in the $2003 R 8$ cultivar is fully functional and the rooting differences observed are due to differential auxin homeostasis between the two cultivars studied. We also found that exogenous auxin treatment did not significantly affect the expression of the EGs encoding putative ARF transcription factors, which also showed similar expression profiles in these two cultivars (Fig. 5b). Our gene expression profiling results are a starting point to identify which auxin response modules involving specific Aux/IAA corepressors and ARF transcription factors are controlling the early steps of AR development in carnation stem cuttings (Fig. 9b). 
As previously found during AR development in Pinus contorta hypocotyls [21], several integral components of the photosynthetic machinery were downregulated during the initial stages of adventitious rooting and up to 54 hAP (Fig. 9a). This clearly shows that cells within the stem cutting base transiently lack their potential to function as photosynthetic cells, which we believe might be linked to the establishment of a new sink within the stem cutting base, as has been described in petunia cuttings [14]. In line with this hypothesis, we found that the expression of genes encoding sucrose degradation enzymes, such as vacuolar invertase (Dca8627 and Dca54544) and cell-wall localized invertase (Dca51558 and Dca59840), showed a biphasic response during adventitious rooting, coinciding with the onset of the induction phase. In addition, Dca4507 encoding a homolog of the Arabidopsis SUCROSE SYNTHASE4 [52] was found upregulated after $6 \mathrm{hAP}$. Our previous results $[15,43]$ indicated a high energy requirement during rooting in the base of the stem, which was provided by an increase of sucrolytic enzymes during the early phases of rooting. With this study, we confirm that the burst of sucrolytic enzymatic activity observed previously is regulated at the transcript level.

Both the histological analysis and the transcript profiling presented in this work confirmed that the timing for the activation of cell division in the cambial initials depended on the cultivar and it was accelerated by the exogenous auxin treatment. In plants, D-type and A3type cyclins have been implicated in the G1-to-S transition $[53,54]$ while subgroups of A-type and B-type cyclins act in the G2-to-M transition $[55,56]$. A number of genes encoding mitotic A-type (Dca24345 and Dca44777) and B-type cyclins (Dca14212, Dca43894 and Dca44193) were clearly upregulated in both cultivars from 24 hAP onwards and their early expression was slightly higher in the auxin-treated samples. These results are in agreement with those found in Arabidopsis, where the cyclin-dependent kinase activity required for mitosis is regulated by redundant genes encoding CYCLINA2 and CYCLINB [55, 57].

Another of the functional groups that showed differences in their expression levels during rooting were those encoding transcripts related to microtubules (MTs) and MT-associated proteins, such as kinesins. MTs play essential roles in cell division and cell elongation [58] and they indirectly might regulate morphogenesis [59]. Several genes encoding MT-associated proteins showed a biphasic response along the time-course experiment with a clear downregulation at earlier stages and a concomitant upregulation at later time points (Fig. 9a and data not shown).

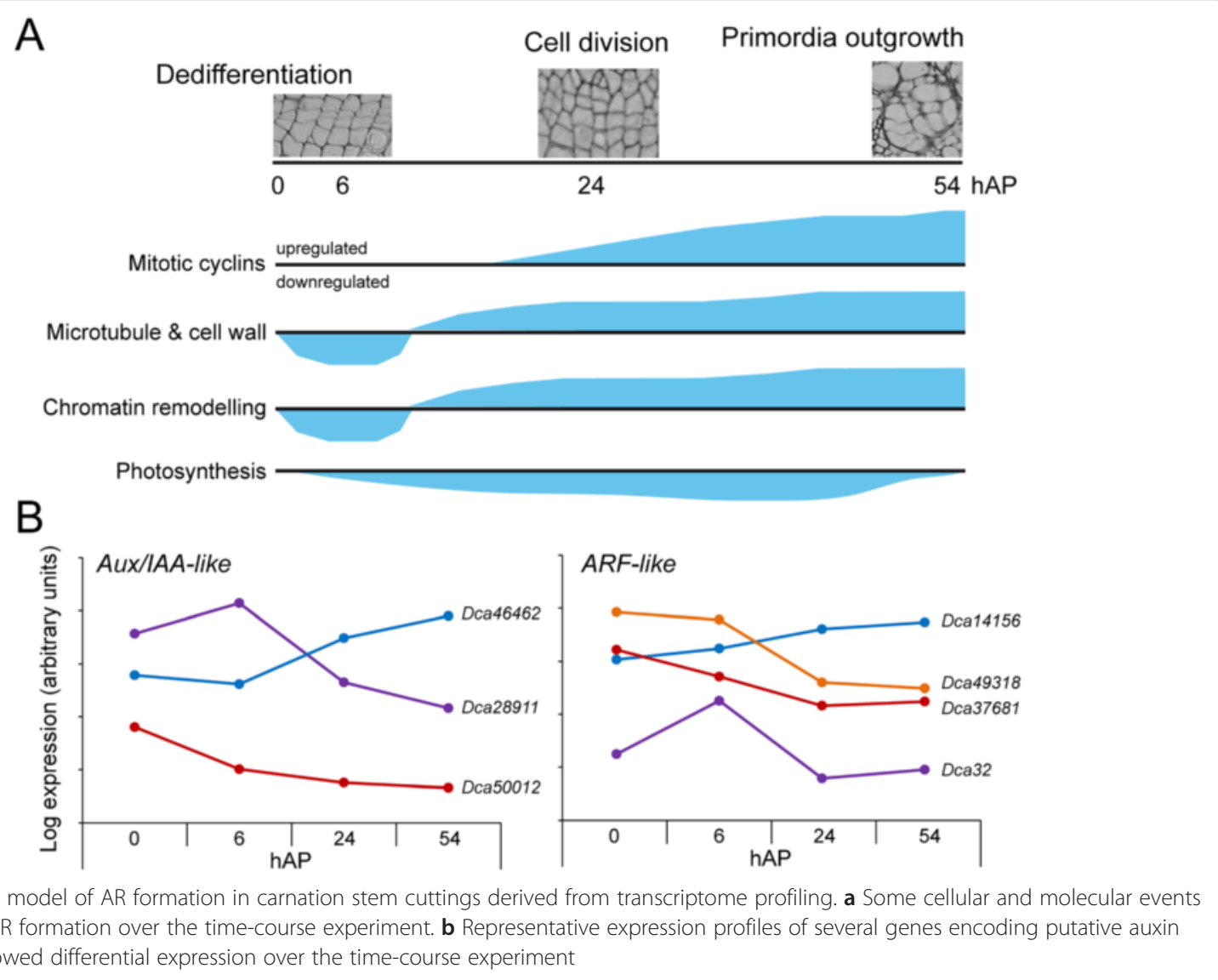


Among those, several kinesin-encoding genes (Dca24841, Dca27864 and Dca45361) were found highly expressed from 24 hAP onwards, coinciding with the activation of cell division. Differential remodelling of MTs has been observed previously in juvenile cuttings compared to mature cuttings in Eucalyptus grandis [23]. If MT dynamics also plays a role in AR formation in carnation, we expect that subtle perturbations of MTs might improve the rooting success of the bad-rooting carnation cultivars, as it was previously shown for mature E. grandis cuttings [23].

Interestingly, we found several genes encoding specific histone variants (Dca5695, Dca16479 and Dca21788) that displayed a biphasic expression profile during adventitious rooting consisting of a slight downregulation during the initial stages (up to $6 \mathrm{hAP}$ ) and a concomitant increase in their expression levels afterwards. A similar expression profile was found for Dca58880, encoding an homolog of the KRYPTONITE (KYP, also known as SUVH4) histone H3 lysine 9 methyltransferase [60]. Recent studies in the Arabidopsis thaliana model indicated that H3.3 (whose putative carnation homolog encoding gene was Dca21788) was associated with active genes and showed a positive correlation with their expression levels, suggesting that $\mathrm{H} 3$ variant replacement may contribute to enable reprogramming at developmental transitions [61]. In addition, the functional loss of KYP resulted in altered expression of developmental regulators, such as WUSCHEL, and defects in callus formation during hormone-mediated dedifferentiation $[62,63]$. Our results suggest that extensive chromatin remodelling is taking place in the stem cutting base in carnation cuttings prior to the activation of cell division. Whether these chromatin regulators are regulated by the inductive (auxin) signal remains to be elucidated.

\section{Conclusions}

With this work we initiated a multidimensional approach to characterize AR formation in the stem cutting base of a series of carnation cultivars with contrasting rooting performance. Our results allowed us to precisely define the different stages during AR formation and to identify a number of molecular, histological and physiological markers. These will allow us to monitor adventitious rooting in a wide collection of carnation germplasm and to select the good-rooting cultivars for breeding purposes.

\section{Availability of supporting data}

RNA-Seq data supporting this study are available in the ArrayExpress database (www.ebi.ac.uk/arrayexpress) under accession number E-MTAB-3698.

\section{Additional files}

\begin{abstract}
Additional file 1: Figure S1. Exploratory data analysis. (A) Heat map representation of the Euclidean distance between samples. The colour code in the histogram goes from white (lowest correlation values) to dark blue (highest correlation values). (B) PCA analysis. (TIFF 2974 kb)
\end{abstract}

Additional file 2: Table S1. Oligonucleotides used in this work. (DOC $30 \mathrm{~kb}$ )

Additional file 3: Data file for evidence-based gene models used in this work. (7Z $1766 \mathrm{~kb}$ )

Additional file 4: Figure S2. Validation of RNA-seq results by qRT-PCR. The relative expression of six genes was studied in the $2003 R 8(\mathrm{~A})$ and 2101-02 MFR (B) cultivars. Each dot represents the relative expression data for a given sample. (TIFF $918 \mathrm{~kb}$ )

Additional file 5: Figure S3. Model profiles comparison between mock-treated and auxin-treated samples in the 2101-02 MFR cultivar. A profile to the immediate left of a yellow bar is from mock-treated samples. A profile to the right of the yellow bar is from the auxin-treated experiment, and has a significant intersection (in terms of the genes assigned to them) with the profile to the left of the yellow bar in its row. The number of genes and the P-value of the intersections are shown in the bottom left-hand. DD, DU, UD and UU are defined in Figure 4. The number on the right, indicates the overlap (in \%) between genes assigned to each profile in mock- and auxin-treated samples. Profiles are coloured by default. (TIFF 3893 kb)

Additional file 6: Figure S4. Cellular parameters in the cambial cells during AR formation. (A) A representative cross-section image of the stem cutting base used to quantify some cellular parameters within the cambium. Squares represent the area measured for disorganized and organized regions. (B) Division rate of the cambial cells in the studied cultivars. Treatments and time-points are represented by coloured bars (white: mock 0 hAP; light grey: mock 54 hAP; dark grey: auxin 0 hAP; black: auxin 54 h AP). Different letters indicate significant differences $(P<0.005)$ between regions. Error bars indicate the standard deviation (SD) for the mean data shown. (C) Number of cambial cells per $\mathrm{mm}^{2}$ in the studied cultivars. Organized and disorganized regions within the cambium are represented as lined and dotted bars, respectively. Asterisks indicate significant differences $(P<0.005)$ between regions at a given time-point. Different letters indicate significant differences $(P<0.005)$ between samples. (TIFF $6084 \mathrm{~kb}$ )

\section{Abbreviations}

ACC: 1-aminocyclopropane-1-carboxylic acid; AR: Adventitious root; Arabidopsis: Arabidopsis thaliana; ARF: AUXIN RESPONSE FACTOR; bp: base pair; BP: Biological process; CC: Cellular component; cDNA: complementary DNA; DAP: Days after planting; DD: DownDown; DEG: Differentially expressed gene; DNA: Deoxyribonucleic acid; DU: DownUp; EGs: Genes with dynamic expression profiles; GO: Gene ontology; GRETCHEN HAGEN3: GH3; hAP: hours after planting; IAA: indole-3-acetic acid; IBA: indole-3-butyric acid; MF: molecular function; miRNAs: microRNAs; MT: microtubules; NAA: a-naphtalene acetic acid; $\mathrm{OBH}$ : one-way best-hits; ORF: open reading frame; PCA: principal-component analysis; qRT-PCR: quantitative reverse-transcription PCR; RNA: ribonucleic acid; RNA-Seq: RNA sequencing; TAIR: The Arabidopsis Information Resource; TF: transcription factor; tZ: trans-zeatin; UD: UpDown; UU: UpUp; WN: Weight/ volume.

\section{Competing interests}

The authors declare that they have no competing interests.

\section{Authors' contributions}

CVM carried out statistical and bioinformatic analyses and helped drafting the manuscript. ABSG performed the validation of the experiments and the histology labwork and participated in drafting the manuscript, JV carried out the treatments in the lab and participated in drafting the manuscript, AC and MA designed and performed the phytohormone analyses, MvdR carried out the library preparations and participated in the coordination of the study, JdH and PP participated in the conception, design and coordination of the study, JMPP conceived the study, collaborated in the time-series analysis and drafted the manuscript. All authors read and approved the final manuscript. 


\section{Acknowledgements}

We are especially indebted to Drs. A. Albacete and F. Pérez-Alfocea (CEBASCSIC, Murcia, Spain) for hormone analyses, and Emilio Á. Cano (Barberet \& Blanc S.A., part of Dümmen Orange) for plant material. This work was supported by the Netherlands Enterprise Agency and the Center for the Development of Industrial Technology of Spain (CARNOMICS Eurostars-EUREKA Project E! 6384), by the Ministerio de Economía y Competitividad of Spain and by FEDER Funds of the European Commission (grant no. AGL2012-33610).

\section{Author details}

'Genetwister Technologies B.V., P.O. Box 193, NL6700 AD Wageningen, The Netherlands. ${ }^{2}$ Instituto de Bioingeniería, Universidad Miguel Hernández, 03202 Elche, Alicante, Spain. ${ }^{3}$ Departamento de Biología Vegetal (Fisiología Vegetal), Universidad de Murcia, Murcia, Spain.

\section{Received: 1 June 2015 Accepted: 3 October 2015}

Published online: 14 October 2015

\section{References}

1. Geiss G, Gutierrez L, Bellini C. Adventitious root formation: New insights and perspectives. In: Beekman T, editor. Root Development. Oxford: Wiley-Blackwell; 2009. p. 376.

2. Bellini C, Pacurar DI, Perrone I. Adventitious roots and lateral roots: similarities and differences. Annu Rev Plant Biol. 2014;65:639-66.

3. de Klerk GJ, Van der Krieken W, de Jong JC. The formation of adventitious roots: new concepts, new possibilities. In Vitro Cell Dev Biol Plant. 1999;35(3):189-99. \%! The formation of adventitious roots: new concepts, new possibilities\%@ 10545476.

4. Pacurar DI, Perrone I, Bellini C. Auxin is a central player in the hormone cross-talks that control adventitious rooting. Physiol Plant. 2014;151(1):83-96.

5. Ahkami AH, Melzer M, Ghaffari MR, Pollmann S, Ghorbani Javid M, Shahinnia $F$, et al. Distribution of indole-3-acetic acid in Petunia hybrida shoot tip cuttings and relationship between auxin transport, carbohydrate metabolism and adventitious root formation. Planta. 2013;238(3):499-517.

6. Garrido G, Guerrero JR, Cano EA, Acosta M, Sánchez-Bravo J. Origin and basipetal transport of the IAA responsible for rooting of carnation cuttings. Physiol Plant. 2002;114(2):303-12.

7. Boerjan W, Cervera MT, Delarue M, Beeckman T, Dewitte W, Bellini C, et al. Superroot, a recessive mutation in Arabidopsis, confers auxin overproduction. Plant Cell. 1995;7(9):1405-19.

8. Delarue M, Prinsen E, Onckelen HV, Caboche M, Bellini C. Sur2 mutations of Arabidopsis thaliana define a new locus involved in the control of auxin homeostasis. Plant J. 1998;14(5):603-11.

9. Zhao Y, Christensen SK, Fankhauser C, Cashman JR, Cohen JD, Weigel D, et al. A role for flavin monooxygenase-like enzymes in auxin biosynthesis. Science. 2001;291(5502):306-9.

10. Rasmussen A, Mason MG, De Cuyper C, Brewer PB, Herold S, Agusti J, et al. Strigolactones suppress adventitious rooting in Arabidopsis and pea. Plant Physiol. 2012;158(4):1976-87.

11. Riefler M, Novak O, Strnad M, Schmulling T. Arabidopsis cytokinin receptor mutants reveal functions in shoot growth, leaf senescence, seed size, germination, root development, and cytokinin metabolism. Plant Cell. 2006;18(1):40-54.

12. Werner T, Motyka V, Laucou V, Smets R, Van Onckelen H, Schmulling T. Cytokinin-deficient transgenic Arabidopsis plants show multiple developmental alterations indicating opposite functions of cytokinins in the regulation of shoot and root meristem activity. Plant Cell. 2003;15(11):2532-50.

13. Ramírez-Carvajal GA, Morse AM, Dervinis C, Davis JM. The cytokinin type-B response regulator PtRR13 is a negative regulator of adventitious root development in Populus. Plant Physiol. 2009;150(2):759-71.

14. Ahkami AH, Lischewski S, Haensch KT, Porfirova S, Hofmann J, Rolletschek H, et al. Molecular physiology of adventitious root formation in Petunia hybrida cuttings: involvement of wound response and primary metabolism. New Phytol. 2009;181(3):613-25.

15. Agulló-Antón MÁ, Sánchez-Bravo J, Acosta M, Druege U. Auxins or Sugars: What Makes the Difference in the Adventitious Rooting of Stored Carnation Cuttings? 2011.

16. Sorin C, Bussell JD, Camus I, Ljung K, Kowalczyk M, Geiss G, et al. Auxin and light control of adventitious rooting in Arabidopsis require ARGONAUTE1. Plant Cell. 2005;17(5):1343-59.
17. Gutierrez L, Bussell JD, Pacurar DI, Schwambach J, Pacurar M, Bellini C. Phenotypic plasticity of adventitious rooting in Arabidopsis is controlled by complex regulation of AUXIN RESPONSE FACTOR transcripts and microRNA abundance. Plant Cell. 2009;21(10):3119-32.

18. Sorin C, Negroni L, Balliau T, Corti H, Jacquemot MP, Davanture M, et al. Proteomic analysis of different mutant genotypes of Arabidopsis led to the identification of 11 proteins correlating with adventitious root development. Plant Physiol. 2006;140(1):349-64.

19. Gutierrez L, Mongelard G, Flokova K, Pacurar DI, Novak O, Staswick P, et al. Auxin controls Arabidopsis adventitious root initiation by regulating jasmonic acid homeostasis. Plant Cell. 2012;24(6):2515-27.

20. Wei K, Wang LY, Wu LY, Zhang CC, Li HL, Tan LQ, et al. Transcriptome analysis of indole-3-butyric acid-induced adventitious root formation in nodal cuttings of Camellia sinensis (L.). PLoS One. 2014;9(9):e107201.

21. Brinker M, van Zyl L, Liu W, Craig D, Sederoff RR, Clapham DH, et al. Microarray analyses of gene expression during adventitious root development in Pinus contorta. Plant Physiol. 2004;135(3):1526-39.

22. Rigal A, Yordanov YS, Perrone I, Karlberg A, Tisserant E, Bellini C, et al. The AINTEGUMENTA LIKE1 homeotic transcription factor PtAIL1 controls the formation of adventitious root primordia in poplar. Plant Physiol. 2012;160(4):1996-2006

23. Abu-Abied M, Szwerdszarf D, Mordehaev I, Yaniv Y, Levinkron S, Rubinstein $M$, et al. Gene expression profiling in juvenile and mature cuttings of Eucalyptus grandis reveals the importance of microtubule remodeling during adventitious root formation. BMC Genomics. 2014;15:826.

24. Ahkami A, Scholz U, Steuernagel B, Strickert M, Haensch KT, Druege U, et al. Comprehensive transcriptome analysis unravels the existence of crucial genes regulating primary metabolism during adventitious root formation in Petunia hybrida. PLoS One. 2014;9(6):e100997.

25. Han H, Sun X, Xie Y, Feng J, Zhang S. Transcriptome and proteome profiling of adventitious root development in hybrid larch (Larix kaempferi $\times$ Larix olgensis). BMC Plant Biol. 2014;14:305.

26. Birlanga V, Villanova J, Cano A, Cano EA, Acosta M, Pérez-Pérez JM Quantitative analysis of adventitious root growth phenotypes in carnation stem cuttings. PLoS One. 2015;10(7):e0133123

27. Yagi M, Kosugi S, Hirakawa H, Ohmiya A, Tanase K, Harada T, et al. Sequence analysis of the genome of carnation (Dianthus caryophyllus L.). DNA Res. 2014;21(3):231-41.

28. Grabherr MG, Haas BJ, Yassour M, Levin JZ, Thompson DA, Amit I, et al. Full-length transcriptome assembly from RNA-Seq data without a reference genome. Nat Biotechnol. 2011;29(7):644-52.

29. Haas BJ, Papanicolaou A, Yassour M, Grabherr M, Blood PD, Bowden J, et al. De novo transcript sequence reconstruction from RNA-seq using the Trinity platform for reference generation and analysis. Nat Protoc. 2013;8(8):1494-512.

30. Ashburner M, Ball CA, Blake JA, Botstein D, Butler H, Cherry JM, et al. Gene ontology: tool for the unification of biology. The Gene Ontology Consortium. Nat Genet. 2000;25(1):25-9.

31. Finn RD, Bateman A, Clements J, Coggill P, Eberhardt RY, Eddy SR, et al. Pfam: the protein families database. Nucleic Acids Res. 2014;42(Database issue):D222-230

32. Finn RD, Clements J, Eddy SR. HMMER web server: interactive sequence similarity searching. Nucleic Acids Res. 2011;39(Web Server issue):W29-37.

33. Falda M, Toppo S, Pescarolo A, Lavezzo E, Di Camillo B, Facchinetti A, et al. Argot2: a large scale function prediction tool relying on semantic similarity of weighted Gene Ontology terms. BMC Bioinform. 2012;13 Suppl 4:S14.

34. Lamesch P, Berardini TZ, Li D, Swarbreck D, Wilks C, Sasidharan R, et al. The Arabidopsis Information Resource (TAIR): improved gene annotation and new tools. Nucleic Acids Res. 2012;40(Database issue):D1202-1210.

35. Love MI, Huber W, Anders S. Moderated estimation of fold change and dispersion for RNA-seq data with DESeq2. Genome Biol. 2014;15(12):550.

36. Bourgon R, Gentleman R, Huber W. Independent filtering increases detection power for high-throughput experiments. Proc Natl Acad Sci U S A. 2010;107(21):9546-51.

37. Robasky K, Lewis NE, Church GM. The role of replicates for error mitigation in next-generation sequencing. Nat Rev Genet. 2014;15(1):56-62.

38. Alexa A, Rahnenfuhrer J. topGO: topGO: Enrichment Analysis for Gene Ontology. In: R package versión 2180. 2.18 .0 edn; 2010: R package.

39. Livak KJ, Schmittgen TD. Analysis of relative gene expression data using real-time quantitative PCR and the 2(-Delta Delta C(T)) Method. Methods. 2001;25(4):402-8. 
40. Ernst J, Bar-Joseph Z. STEM: a tool for the analysis of short time series gene expression data. BMC Bioinform. 2006;7:191.

41. Großkinsky DK, Albacete A, Jammer A, Krbez P, van der Graaff E, Pfeifhofer $\mathrm{H}$, et al. A rapid phytohormone and phytoalexin screening method for physiological phenotyping. Mol Plant. 2014;7(6):1053-6.

42. Guo A, He K, Liu D, Bai S, Gu X, Wei L, et al. DATF: a database of Arabidopsis transcription factors. Bioinformatics. 2005;21(10):2568-9.

43. Agulló-Antón MA, Ferrández-Ayela A, Fernández-García N, Nicolás C, Albacete A, Pérez-Alfocea F, et al. Early steps of adventitious rooting: morphology, hormonal profiling and carbohydrate turnover in carnation stem cuttings. Physiol Plant. 2014;150(3):446-62.

44. Cano A, Pérez-Pérez JM, Acosta M. Adventitious Root Development in Ornamental Plants: Insights from Carnation Stem Cuttings, vol. 40. Biology: Springer-Verlag Berlin Heidelberg; 2014

45. Negi S, Sukumar P, Liu X, Cohen JD, Muday GK. Genetic dissection of the role of ethylene in regulating auxin-dependent lateral and adventitious root formation in tomato. Plant J. 2010;61(1):3-15.

46. Druege U, Franken P, Lischewski S, Ahkami AH, Zerche S, Hause B, et al. Transcriptomic analysis reveals ethylene as stimulator and auxin as regulator of adventitious root formation in petunia cuttings. Front Plant Sci. 2014;5:494.

47. Galinha C, Hofhuis H, Luijten M, Willemsen V, Blilou I, Heidstra R, et al. PLETHORA proteins as dose-dependent master regulators of Arabidopsis root development. Nature. 2007:449(7165):1053-7.

48. Rasmussen A, Smith T, Hunt M. Cellular stages of root formation, root system quality and survival of Pinus. 2009

49. Tian $\mathrm{Q}$, Reed J. Control of auxin-regulated root development by the Arabidopsis thaliana SHY2/IAA3 gene. Development. 1999;126(4):711-21.

50. Tatematsu K, Kumagai S, Muto H, Sato A, Watahiki MK, Harper RM, et al. MASSUGU2 encodes Aux/IAA19, an auxin-regulated protein that functions together with the transcriptional activator NPH4/ARF7 to regulate differential growth responses of hypocotyl and formation of lateral roots in Arabidopsis thaliana. Plant Cell. 2004;16(2):379-93.

51. Fukaki H, Tameda S, Masuda H, Tasaka M. Lateral root formation is blocked by a gain-of-function mutation in the SOLITARY-ROOT/IAA14 gene of Arabidopsis. Plant J. 2002;29(2):153-68.

52. Bieniawska Z, Paul Barratt DH, Garlick AP, Thole V, Kruger NJ, Martin C, et al. Analysis of the sucrose synthase gene family in Arabidopsis. Plant J. 2007:49(5):810-28.

53. Dewitte W, Scofield S, Alcasabas AA, Maughan SC, Menges M, Braun N, et al. Arabidopsis CYCD3 D-type cyclins link cell proliferation and endocycles and are rate-limiting for cytokinin responses. Proc Natl Acad Sci U S A. 2007:104(36):14537-42

54. Takahashi I, Kojima S, Sakaguchi N, Umeda-Hara C, Umeda M. Two Arabidopsis cyclin A3s possess G1 cyclin-like features. Plant Cell Rep. 2010;29(4):307-15.

55. Schnittger A, Schöbinger U, Stierhof YD, Hülskamp M. Ectopic B-type cyclin expression induces mitotic cycles in endoreduplicating Arabidopsis trichomes. Curr Biol. 2002;12(5):415-20.

56. Boudolf V, Lammens T, Boruc J, Van Leene J, Van Den Daele H, Maes S, et al. CDKB1;1 forms a functional complex with CYCA2;3 to suppress endocycle onset. Plant Physiol. 2009;150(3):1482-93.

57. Vanneste S, Coppens F, Lee E, Donner TJ, Xie Z, Van Isterdael G, et al. Developmental regulation of CYCA2s contributes to tissue-specific proliferation in Arabidopsis. EMBO J. 2011;30(16):3430-41.

58. Wasteneys GO, Fujita M. Establishing and maintaining axial growth: wall mechanical properties and the cytoskeleton. J Plant Res. 2006;119(1):5-10.

59. Landrein B, Hamant O. How mechanical stress controls microtubule behavior and morphogenesis in plants: history, experiments and revisited theories. Plant J. 2013;75(2):324-38.

60. Jackson JP, Lindroth AM, Cao X, Jacobsen SE. Control of CpNpG DNA methylation by the KRYPTONITE histone $\mathrm{H} 3$ methyltransferase. Nature. 2002:416(6880):556-60.

61. Wollmann $H$, Holec S, Alden $K$, Clarke ND, Jacques P, Berger F. Dynamic deposition of histone variant $\mathrm{H} 3.3$ accompanies developmental remodeling of the Arabidopsis transcriptome. PLoS Genet. 2012;8(5):e1002658.

62. Grafi G, Ben-Meir H, Avivi Y, Moshe M, Dahan Y, Zemach A. Histone methylation controls telomerase-independent telomere lengthening in cells undergoing dedifferentiation. Dev Biol. 2007;306(2):838-46.

63. Li W, Liu H, Cheng ZJ, Su YH, Han HN, Zhang Y, et al. DNA methylation and histone modifications regulate de novo shoot regeneration in Arabidopsis by modulating WUSCHEL expression and auxin signaling. PLOS Genet. 2011;7(8):e1002243

\section{Submit your next manuscript to BioMed Central and take full advantage of:}

- Convenient online submission

- Thorough peer review

- No space constraints or color figure charges

- Immediate publication on acceptance

- Inclusion in PubMed, CAS, Scopus and Google Scholar

- Research which is freely available for redistribution 